\title{
Lights on 2,5-diaryl tetrazoles: applications and limits of a versatile photoclick reaction
}

\author{
Valentina Pirota ${ }^{1} \cdot$ Alessandra Benassi $^{1} \cdot$ Filippo Doria $^{1}$ (i)
}

Received: 26 October 2021 / Accepted: 13 January 2022 / Published online: 21 February 2022

(C) The Author(s) 2022

\begin{abstract}
Recently, photoclick chemistry emerged as a powerful tool employed in several research fields, from medicinal chemistry and biology to material sciences. The growing interest in this type of chemical process is justified by the possibility to produce complex molecular systems using mild reaction conditions. However, the elevated spatio-temporal control offered by photoclick chemistry is highly intriguing, as it expands the range of applications. In this context, the light-triggered reaction of 2,5-diaryl tetrazoles with dipolarophiles emerged for its interesting features: excellent stability of the substrates, fast reaction kinetic, and the formation of a highly fluorescent product, fundamental for sensing applications. In the last years, 2,5-diaryl tetrazoles have been extensively employed, especially for bioorthogonal ligations, to label biomolecules and nucleic acids. In this review, we summarized recent applications of this interesting photoclick reaction, with a particular focus on biological fields. Moreover, we described the main limits that affect this system and current strategies proposed to overcome these issues. The general discussion here presented could prompt further optimization of the process and pave the way for the development of new original structures and innovative applications.
\end{abstract}

\section{Graphical abstract}

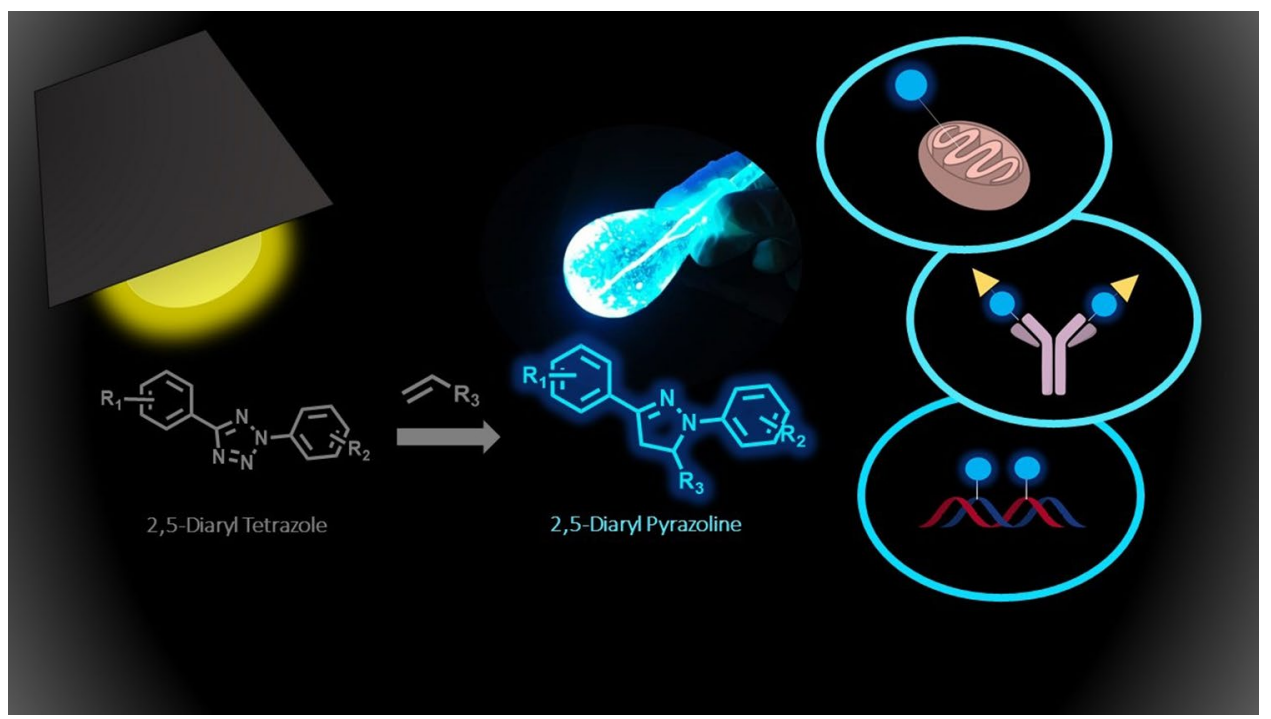

Keywords Tetrazoles $\cdot$ Photoclick reaction $\cdot$ Bioorthogonal chemistry $\cdot$ Fluorescent probes $\cdot$ Cycloaddition

\author{
Alessandra Benassi \\ alessandra.benassi01@universitadipavia.it \\ $\triangle$ Filippo Doria \\ filippo.doria@unipv.it
}

Extended author information available on the last page of the article 


\section{Introduction}

Over the years, click chemistry emerged as a powerful tool that found applications in different research fields, from material science to pharmacology $[1,2]$. The concept of "click reaction" has been introduced in 2002, when Sharpless et al. proposed the use of Huisgen 1,3-dipolar cycloaddition, among an azide and an alkyne, to give 1,2,3-triazoles [3], to assemble in situ potent inhibitors of acetylcholinesterase [4]. The choice of this reaction was prompted by the necessity to operate under physiological conditions, which require specific features: (1) the process has to be operationally simple and applicable to highly diversified substrates; (2) the product formation has to be fast and occur with high yield and selectivity; (3) the reaction must lead to the generation of a single product; (4) reaction conditions should be bio-compatible; (5) the involved compounds have to be non-toxic and stable in the physiological environment [5].

During the years, click reactions found application in several research fields, as in organic synthesis [6], in nanoparticle development [7], as well as in the biomedical field. Regarding the latter, they have been widely used for bioorthogonal ligations [8], needed for imaging of biomolecules, drug delivery, diagnostic analysis [9] as well as for the detection of proteins [10] and nucleic acids [11]. Together with Huisgen cycloaddition, several click reactions have been developed and optimized, as Staudinger ligation [12], the strain-promoted azide-alkyne cycloaddition [13], Inverse-electron demanded Diels-Alder among tetrazines and alkenes [14], and condensation with 2-acylboronic acid [15]. Nevertheless, one of the major drawback shared by these reactions is the impossibility to control the overall process, which can occur in a non-selective manner and lead to undesired secondary reactions. In this context, photochemistry resulted to be the most promising solution to overcome this limitation. Light offers several advantages, including mild reaction conditions and an elevated spatio-temporal control of the process [16]. Recently, several photoclick reactions have been engineered to this purpose, as light-activable hetero Diels-Alder [17], azide-alkyne cycloadditions [18], lighttriggered sydnone-alkene/alkyne cycloaddition [19], photoactivable azirines [20], and diazirines [21], extensively described in recent review articles [16, 22]. In this pool, the photoclick reaction of 2,5-diaryl tetrazoles emerged for its interesting properties [22]. Irradiation of these substrates with a low-power light source induces tetrazole photolysis, generating a 1,3-nitrile imine dipole (NI) which can react with alkenes, affording 2,5-diaryl pyrazolines. One of the most intriguing feature of this reaction is the generation of fluorogenic molecules, which facilitates the monitoring of the process in situ [23]. In addition, considering that alkene derivatives can be easily incorporated into biological systems, this photoclick reaction lends itself to multiple applications [24].

Despite the growing interest in the light-triggered fluorogenic cycloaddition of 2,5-diaryl tetrazoles, to the best of our knowledge, a general overview exclusively dedicated to 2,5-diaryl tetrazole reactivity and their principal biological applications has never been reported. We believe that this kind of report could be helpful not only to clearly take stock of the current achievements but also to highlight their most significant properties and most critical issues, to prompt the research towards more efficient processes.

In this review, we have summarized the principal applications of this process in the biological field, where they have been extensively used as bioorthogonal reporters to label proteins and nucleic acids or to generate new photoresponsive biomaterials. In the end, we have highlighted some related critical points together with the most recent strategies proposed to overcome these issues.

\section{Photochemical properties of 2,5-diaryl tetrazoles}

Five-membered heterocyclic derivatives, as pyrroles [25], imidazoles [26], triazoles [27, 28], and oxadiazoles [29, 30] attracted increasing attention during the last years for their interesting biological properties [31]. In this context, tetrazoles are a class of non-natural heterocyclic compounds, composed of a five-membered ring, and can be mono- or di-substituted (Fig. 1A). In solution, they can exist in two tautomeric forms, $1 \mathrm{H}$, more stable [32], or $2 \mathrm{H}$ (Fig. 1B). Functionalization in position 5 on the carbon atom is the most common, as empowers the applications of these types of derivatives in biochemistry, pharmacology, material science, photography, and even in the military field [33]. However, their most important use regards pharmacology: currently, 43 drugs contain tetrazole ring, and, among them, 23 are FDA approved, as antimicrobial, antiviral, antiallergic, and anticancer agents [34]. Their excellent biological activity could be partially attributed to their acidic properties $\left(\mathrm{pK}_{\mathrm{a}} \approx 4.5-4.9\right)$, indeed they are widely used as bioisosteres of carboxylic acids [35].

Tetrazoles are particularly interesting scaffolds for their unique photochemical properties: irradiation with UV light always induces rapid irreversible cycloreversion reaction resulting in nitrogen release [36]. In this context, the substitution of the substrate strongly influences photoreactivity, leading to different reaction pathways and products of different nature [37]. Among all, 2,5-diaryl tetrazoles attracted significant attention during the last years, for their photoactivation properties and for the types of products generated. 
Fig. 1 A General structures of different substituted tetrazoles. B $1 \mathrm{H}$ and $2 \mathrm{H}$ tautomeric forms of tetrazoles
A)<smiles>[R]c1nnn[nH]1</smiles>

5-substitued tetrazole<smiles>[R]c1nnnn1[R]</smiles>

1,5-disubstitued tetrazole<smiles>[R]c1nnn([R])n1</smiles>

2,5-disubstitued tetrazole

B)

\begin{tabular}{|c|}
$1 H$-tetrazole \\
2H-tetrazole
\end{tabular}

Moreover, a non-negligible feature of these compounds is their straightforward synthetic procedure: they can be easily generated with a single reaction step, through a simple coupling between benzensulphonyl hydrazone and an arene diazonium salt [38].

Irradiation of tetrazoles, at an appropriate wavelength, leads to the formation of a bidentate intermediate, a 1,3-nitrile imine dipole (Fig. 2), which can react with both nucleophiles [39], and dipolarophiles, especially electronpoor alkenes [40]. Despite a large amount of data about nitrile imine reactivity, the generation mechanism of this intermediate and its electronic properties are still subject to debate. Computational analysis evidenced that the first excited state $S_{1}$ of 2,5-diaryl tetrazoles lies close in energy to the triplet state $\mathrm{T}_{3}$, suggesting that nitrile imine formation occurs via fast intersystem crossing process (ISC) [41]. More recently, activation via triplet state has been detected through ESR analysis in $5 \mathrm{~K}$ matrix, highlighting that the substitution of the scaffold strongly influences the reaction pathway. In the case of 1,5-diaryl tetrazoles, the ISC is followed by the generation of an imidoylnitrene, that rearranges into nitrile imine dipole, while irradiation of 2,5-diaryl tetrazole at $266 \mathrm{~nm}$ with $5 \mathrm{Jm}$ laser pulses, induces ISC, immediately followed by the formation of 1,3-dipolar intermediate

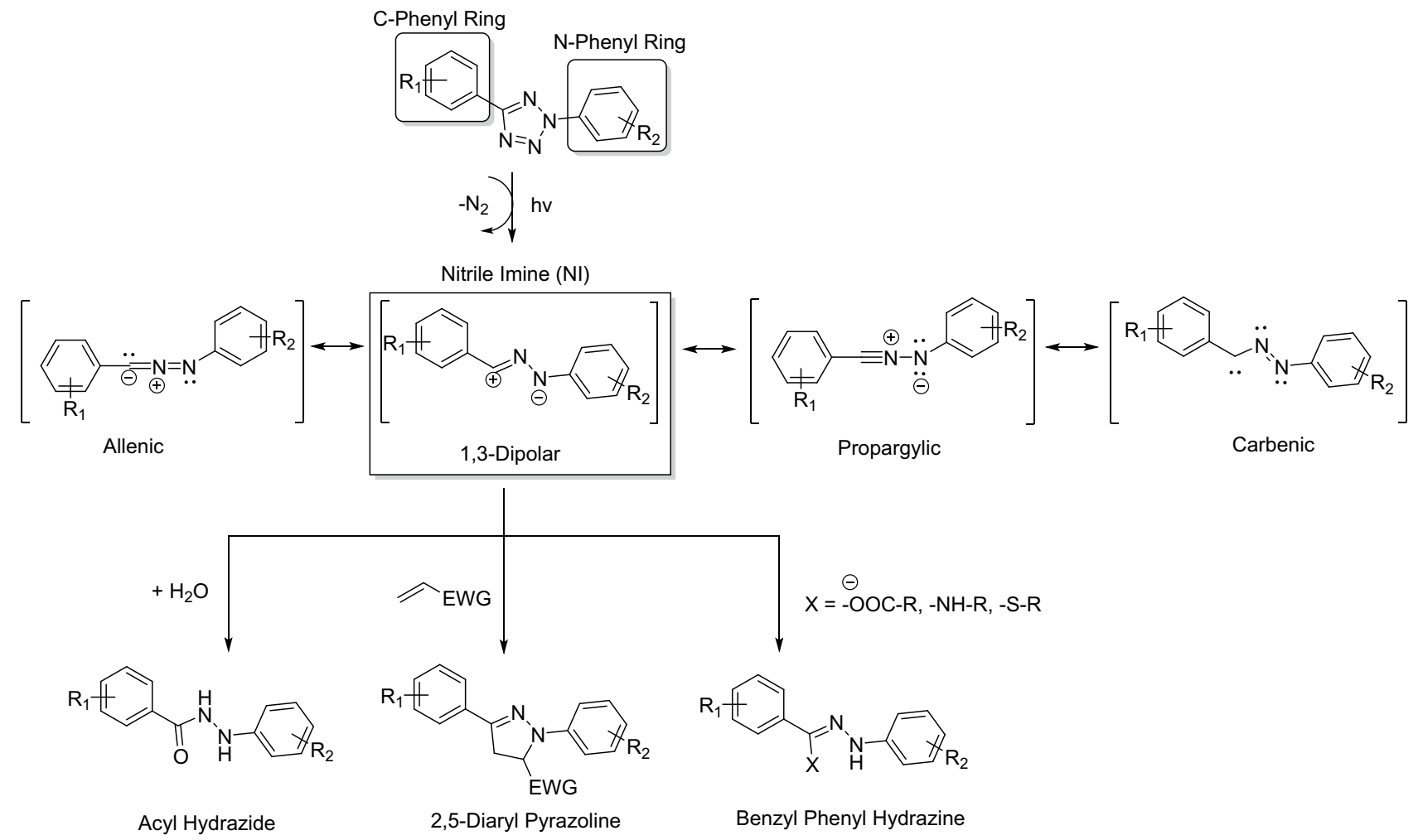

Fig. 2 Photogeneration of 1,3-Nitrile Imine dipole from 2,5-diaryl tetrazoles and possible reaction pathways 
[42]. However, further studies are still required to confirm these results and to obtain more experimental evidences on the tetrazole photolysis mechanism.

The nitrile imine is a very reactive species that cannot be isolated and has been observed only as intermediate using transient spectroscopic techniques [43], so its electronic properties are still the object of investigation. In principle, it has been suggested that nitrile imine could exist in propargylic, allenic [44], 1,3-dipolar, and carbenic forms [45] (Fig. 2). Despite different studies provided contrasting results, a detailed experimental analysis of Lin et al. [46] strongly contributed to the elucidation of the structure of this reactive species. Irradiating the zinc complex of 2-(4'-methoxyphenyl)-5-(2"-isopropoxy-4"'-methoxyphenyl) tetrazole with $325 \mathrm{~nm} \mathrm{He-Cd}$ laser, at $90 \mathrm{~K}$, X-ray analysis evidenced that nitrile imine presented a bent geometry which could be attributed to 1,3-dipolar or carbenic structures. In addition, trapping experiments with water confirmed that nitrile imine primarily exists as 1,3-dipolar structure [46].

This intermediate presents high versatility, due to its electronic properties, and can react with both nucleophiles, like water, thiols, carboxylates, and amines, and electrophiles, especially alkenes, via 1,3-dipolar cycloaddition [47]. This second reaction pathway is the most interesting for biological applications, as it leads to the generation of fluorescent 2,5-diaryl pyrazolines, which can be exploited to localize specific biomolecules and/or monitor physiological processes [48]. However, in the cellular environment, nucleophilic addition competes with the cycloaddition, reducing the selectivity of the process; therefore, to limit the formation of undesired products, the structure of the tetrazole must be properly modified to drive the reactivity of the corresponding intermediate towards the chosen route, as it will be discussed later [49]. The strong potential of this photoclick reaction has been demonstrated first by Lin et al., as they examined the photoreactivity of 2,5-diaryl tetrazole 1 (Fig. 3) under irradiation at $302 \mathrm{~nm}$ in water solution, in presence of acrylamide as a trapping agent of the NI. Tetrazole photolysis was remarkably fast, with first-order rate constant $k_{1}=0.14 \mathrm{~s}^{-1}$, as well as the following cycloaddition with the alkene, which proceeded rapidly, with high yields, and with second-order rate constant $k_{2}=11.0 \mathrm{M}^{-1} \mathrm{~s}^{-1}$. Furthermore, product generation resulted in a marked increase of fluorescence emission, among 487-538 nm, due to the high fluorescence quantum yield of 2,5-diaryl pyrazoline ( $\Phi_{\mathrm{f}}=0.29$ in phosphate buffer solution). The bioorthogonality of the reaction has been tested by conjugating tetrazole $\mathbf{1}$ to Lysozyme and performing photoirradiation at physiological conditions in presence of acrylamide. In only two minutes, $90 \%$ conversion was achieved and formation of the desired fluorescent adduct was observed [50]. These excellent results laid the basis for the subsequent development of photoreactive tetrazole scaffolds for bioorthogonal ligations. However, it has been pointed out that this reaction worked efficiently only in presence of electron-withdrawing alkenes, otherwise both selectivity and reaction rate decrease significantly.

The same authors performed an extensive study on different tetrazole scaffolds, to improve reaction rate and expand the reactivity towards electron-rich dipolarophiles. A library of different substituted 2,5-diaryl tetrazoles has been synthesized, introducing in para and meta positions functional groups with various electronic effects, to verify their influence on cycloaddition rate. Irradiation of compounds in phosphate buffer solution (PBS) and in presence of an excess of 4-penten-1-ol, resulted in different outcomes: in particular, the presence of only one substituent on C-phenyl ring, decreased significantly the cycloaddition rate. The same effect has been obtained by placing an electron-withdrawing substituent in para position of $N$-phenyl ring. Interestingly, electron-donating groups remarkably improved the reaction rate and, in presence of an amino group (as for tetrazole 2 in Fig. 3), the second-order rate constant displayed the highest value $\left(k_{2}=0.79 \mathrm{M}^{-1} \mathrm{~s}^{-1}\right)$ giving rise to a $50 \%$ yield of the desired pyrazoline in only $75 \mathrm{~s}$ of irradiation [49].

The photoreactivity of 2,5-diaryl tetrazoles has been extensively explored in presence of alkenes [40], and more recently, also towards alkynes, to further expand the reaction scope and its applicability. Cycloaddition of 1,3-nitrile imine to triple bonds leads to the formation of

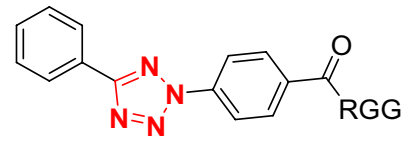

1

RGG = Arg-Gly-Gly-OH

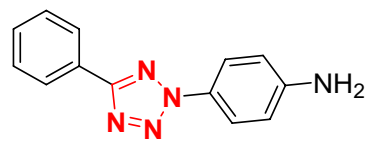

2

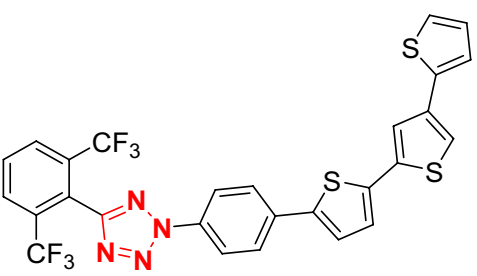

3

Fig. 3 Examples of 2,5-diaryl tetrazoles used for photoreactivity studies 
pyrazoles, interesting scaffolds for their biological properties [51].

The feasibility of this process has been primarily investigated by Bochet et al.,: after the synthesis of several 2,5-substituted tetrazoles, they found that cycloaddition is strictly dependent on the type of electronic properties of the involved substrates. Activation of a non-substituted 2,5-diaryl tetrazole led to the formation of the expected pyrazoline only with electron-poor alkynes: the presence of electron-withdrawing substituents, as esters, nitro, or fluorinated alkyl groups favored the cycloaddition with electron-rich nitrile imine. In addition, it emerged that the reaction occurred also with 3-benzylcyclooctyn, as the elevated ring strain of this substrate promoted the cycloaddition. In presence of these specific types of alkynes, a broad pool of substituted tetrazoles reacted efficiently, generating the desired pyrazole in satisfactory yields (30-99\%) [52].

More recently, Yu et al., further investigated this process and developed a series of 2,5-diaryl tetrazoles containing a trifluoromethyl-phenyl moiety in position 5 and an oligothiophene on $N-2$, to increase both reaction rate and selectivity towards triple bonds. Among tested alkynes, they discovered that the most efficient reaction was obtained using bicyclononyne as a trapping agent. Irradiation of compound 3 (Fig. 3) at 311, 371, and $405 \mathrm{~nm}$ in aqueous solution, led to an almost quantitative yield of the corresponding pyrazole. Furthermore, they have investigated the selectivity of the cycloaddition, performing competition experiments in presence of water and glutathione obtaining the desired pyrazole with excellent yields (89\%) even in these conditions [53]. These intriguing results not only contributed to the clarification of tetrazoles photochemical behavior but represent a solid starting point to expand the applicability of the process.

\section{Biological applications of 2,5-diaryl tetrazoles}

For their excellent optical properties, 2,5-diaryl tetrazoles have found wide application in biological research, in particular as fluorogenic sensors to detect biomolecules in their native environment and to elucidate their biological functions [54]. Initially, they have been extensively used for proteins functionalization, taking advantage of several well-established procedures to introduce non-natural amino acids into their sequences [54] but, more recently, examples of application on nucleic acids and for the development of innovative biomaterials increased significantly.

\subsection{Protein labeling}

The first examples of protein labeling in cells with tetrazoles-based bioorthogonal ligation were reported by Lin and its research group in 2008. They genetically incorporated a modified $O$-allyl tyrosine in the Z-domain of $E$. Coli protein and analyzed pyrazoline formation upon irradiation of a library of tetrazoles with a $302 \mathrm{~nm}$ lamp. Protein labeling proceeded rapidly with compound 4 (Fig. 4); therefore, they tested the efficacy of the process in the cellular environment: E. Coli BL21 (DE3) cells were treated to express $O$-allyl tyrosine-modified protein, then they were incubated with tetrazole 4 and irradiated for $4 \mathrm{~min}$ at $302 \mathrm{~nm}$. The formation of the expected pyrazoline has been detected through fluorescence microscopy, and its emission at $483 \mathrm{~nm}$ was observed only in cells expressing the alkene moiety, evidencing that fluorescence increase was specifically induced by pyrazoline generation [55]. Similarly, another alkenemodified aminoacid, homoallylglycine, has been used for the same purpose: it has been incorporated into the $\beta$-galactosidase enzyme of $293 \mathrm{~T}$ cells, and the efficacy of ligation with BODIPY-modified tetrazole was preliminarily investigated in vitro on cell lysate. Once pyrazoline formation was confirmed, the cycloaddition was performed in live HeLa cells, incubated with tetrazole 2 (Fig. 3), and irradiated with a two-photon $700 \mathrm{~nm}$ laser for $5 \mathrm{~s}$. Only directly illuminated cells showed a 2 -fold increase in fluorescence intensity, confirming the elevated spatio-temporal control offered by this process [56].

Alternatively, the tetrazole unit can be incorporated into the biomolecule and acts as labeling site. To this aim, a library of 2,5-diaryl tetrazoles conjugated to $\alpha$-amino acids has been developed [57] and compound 5 (Fig. 4) was genetically incorporated into Myoglobin of E. Coli, using aminoacyl-tRNA synthetase [58]. To verify the potential labeling of Myoglobin, this was irradiated for $5 \mathrm{~min}$ in presence of fumarate modified with fluorescein isothiocyanate, to ensure efficient product detection. The selective formation of desired product was confirmed by sodium dodecyl sulfate-polyacrylamide gel electrophoresis (SDS-PAGE) and in-gel fluorescence analysis [58]. Despite these promising achievements, incorporation of small-sized moieties (e.g. alkenes) is easier compared to bulkier 2,5-diaryl tetrazoles. Therefore, in general, labeling of proteins is more commonly performed by introducing double bonds on lateral chains of the amino acids. In this scenario, another interesting site for proteins modification, useful for bioorthogonal ligation, is represented by cysteine. Nallani et al., used allyl-cysteine to perform cycloaddition with tetrazole 6 (Fig. 4), introducing this moiety on bovine beta-lactoglobulin. In this case, site-selective functionalization has been achieved, as this protein contains only two cysteines and only one is easily accessible. The photochemical reaction has been carried 
A)
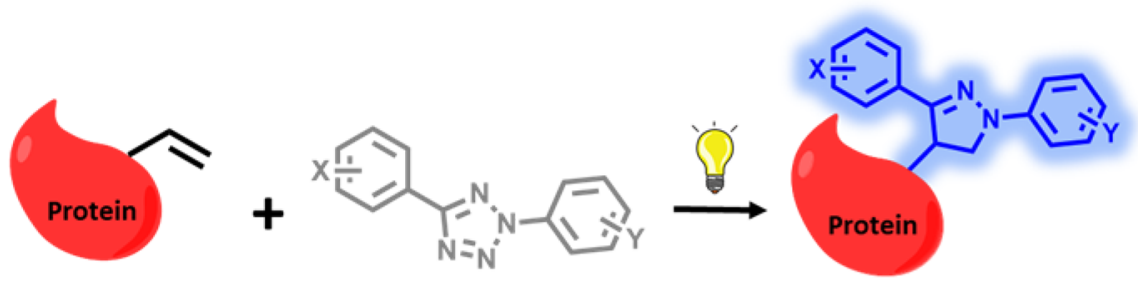

B)

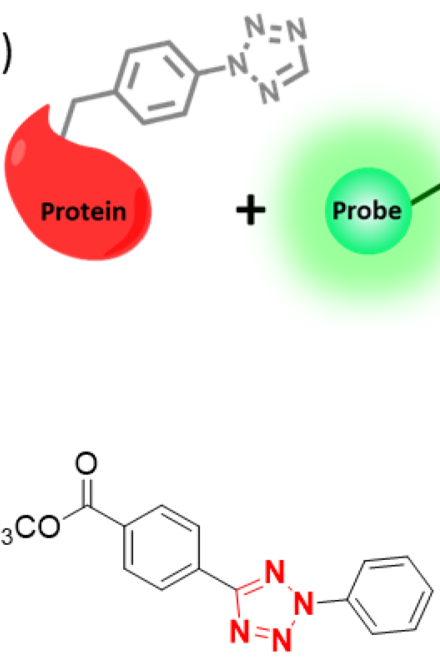

4<smiles>COc1ccc(-n2nnc(-c3ccccc3)n2)cc1</smiles>

7<smiles>NC(Cn1ncnn1)C(=O)O</smiles>
5<smiles>COc1ccc(-n2nnc(-c3c(-c4cccn4C(=O)OC(C)(C)C)cccc3-c3cccn3C(=O)OC(C)(C)C)n2)cc1</smiles><smiles>CCC(CC)(CC)OCCOC(=O)c1ccc(-n2nnc(-c3ccccc3)n2)cc1</smiles><smiles></smiles>

Fig. 4 A Generic photoclick reaction performed with alkene-modified proteins. B Generic photoclick reaction performed with tetrazole-modified proteins. C Examples of 2,5-diaryl tetrazoles used for bioorthogonal labeling of proteins

out in vitro, upon irradiation at $302 \mathrm{~nm}$, for $15 \mathrm{~min}$, and the formation of the corresponding fluorescent product was detected through gel electrophoresis and, subsequently, with MALDI-TOF analysis. Experiments were repeated also on different non-modified proteins/enzymes, like Horseradish Peroxidase, Candida Antarctica lipase, and lysozyme. After 15 min of irradiation, the formation of an adduct with nitrile imine was detected in each case, through mass analysis, and it has been discovered that it was generated by the reaction with tryptophan, which offered an additional and natural site for protein labeling [59]. To increase the fluorescence quantum yield of the generated pyrazoline, and facilitate product detection, styrene has been introduced to modify protein sequence. It has been demonstrated that pyrazoline generated from tetrazole 7 (Fig. 4) and styrene showed significantly higher quantum yield compared to adduct derived from other alkenes $\left(\Phi_{\mathrm{f}}=0.40\right.$ in acetonitrile and $\Phi_{\mathrm{f}}=0.23$ in phosphate buffer solution), with emission at $478 \mathrm{~nm}$. Therefore, styrene has been used to label Acid Stress Chaperone, an intracellular stress-response protein. After incorporation, E. Coli live cells containing protein modified with styrene were incubated with compound $7(100 \mu \mathrm{M})$ and, after only $60 \mathrm{~s}$ of irradiation, a strong fluorescent signal was measured through fluorescence microscopy analysis [60]. To further improve reaction efficiency, bioconjugation has been performed exploiting a rationally designed tetrazole structure. 
Lin's research group modified the substrate scaffold introducing high steric hindrance on the $\mathrm{C}$-phenyl ring: this effect has been achieved by placing two $o-2$ '- $N$-Boc-pyrroles in positions 2 and 6 of the aromatic moiety. The presence of bulky units increased the stability of 1,3-nitrile imine dipole, whose lifetime in water solution increased from 7.5 to $102 \mathrm{~s}$. This remarkable stabilization strongly influenced the selectivity of the process: indeed, upon irradiation of compound 8 (Fig. 4) at $302 \mathrm{~nm}$ in aqueous solution and in presence of glutathione and different alkenes, the reaction resulted to be extremely selective towards the alkene in all tested conditions, even if the best outcome was reached with dimethyl fumarate and trans-cyclooctene. To verify the applicability of this approach, compound $\mathbf{8}$ conjugated to a fluorescent cyanine has been used to label a glucagon receptor (GCGR), which is a common target for the treatment of diabetes, modified with a reactive spiro[2.3] hex-1ene-lysine (SphK). HEK 293 cells expressing the modified GCGR were incubated with $500 \mathrm{nM} 8$ and were irradiated at $302 \mathrm{~nm}$. After only $1 \mathrm{~min}$, fluorescence emission, colocalized with the target receptor, increased significantly (in particular at $\lambda_{\mathrm{em}}=480 \mathrm{~nm}$ ) and pyrazoline was formed with $89 \%$ labeling efficiency, demonstrating the success of this strategy [61]. In this case, an additional fluorescent probe was used to facilitate the detection of product formation, evidencing that sometimes the fluorescence quantum yield of the generated pyrazoline is not sufficiently high to allow efficient detection in a physiological context. To further improve this critical issue, the same authors developed a library of $\mathbf{8}$ analogues, introducing substituents with different electronic effects on $\mathrm{N}$-phenyl ring of the substrate, to evaluate the influence on reaction rate and, most importantly, on fluorescence emission of pyrazoline generated by cycloaddition to $\mathrm{SphK}$. The kinetic analysis confirmed that cycloaddition rate strictly depends on the type of functional group introduced and most rapid reactions occurred in presence of electron-donating moieties on $\mathrm{N}$-2-phenyl ring. Subsequently, they have assessed the effects on fluorescence emission of pyrazolines, discovering that the introduction of $p$-NHAc-Ph group guarantees the most intense emission in organic solvents. This outcome highlighted that this type of functional group could be critical for obtaining both a high reaction rate $\left(k_{2}=1700 \pm 300 \mathrm{M}^{-1} \mathrm{~s}^{-1}\right)$ and intense fluorescence emission ( $\lambda_{\mathrm{em}}=486 \mathrm{~nm}$ in DCM and ethyl acetate). Therefore, this reaction has been exploited to label Green Fluorescent Protein containing SphK. Irradiation of compound 9 (Fig. 4) with $365 \mathrm{~nm}$ UV-light afforded the desired product with good yields and excellent emission in aqueous solution, as confirmed by SDS-PAGE gel analysis [62].

Detection of specific proteins using photoclick chemistry is possible also exploiting an intramolecular cycloaddition. Yu et al., developed a short series of interesting tetrazole derivatives, with $\mathbf{1 0}$ (Fig. 5) as a common unit, introducing $O$-allyloxy group in position 2 of $N$-phenyl ring. In this case, the process has been exploited to detect microtubules in live mammalian cells, which represents an interesting biological target especially for cancer [63] and neurodegenerative disease therapeutic strategies [64]. To this aim, tetrazoles have been conjugated to 7- $\beta$-alanyltaxol core [65], a $\beta$-tubulin binding agent,
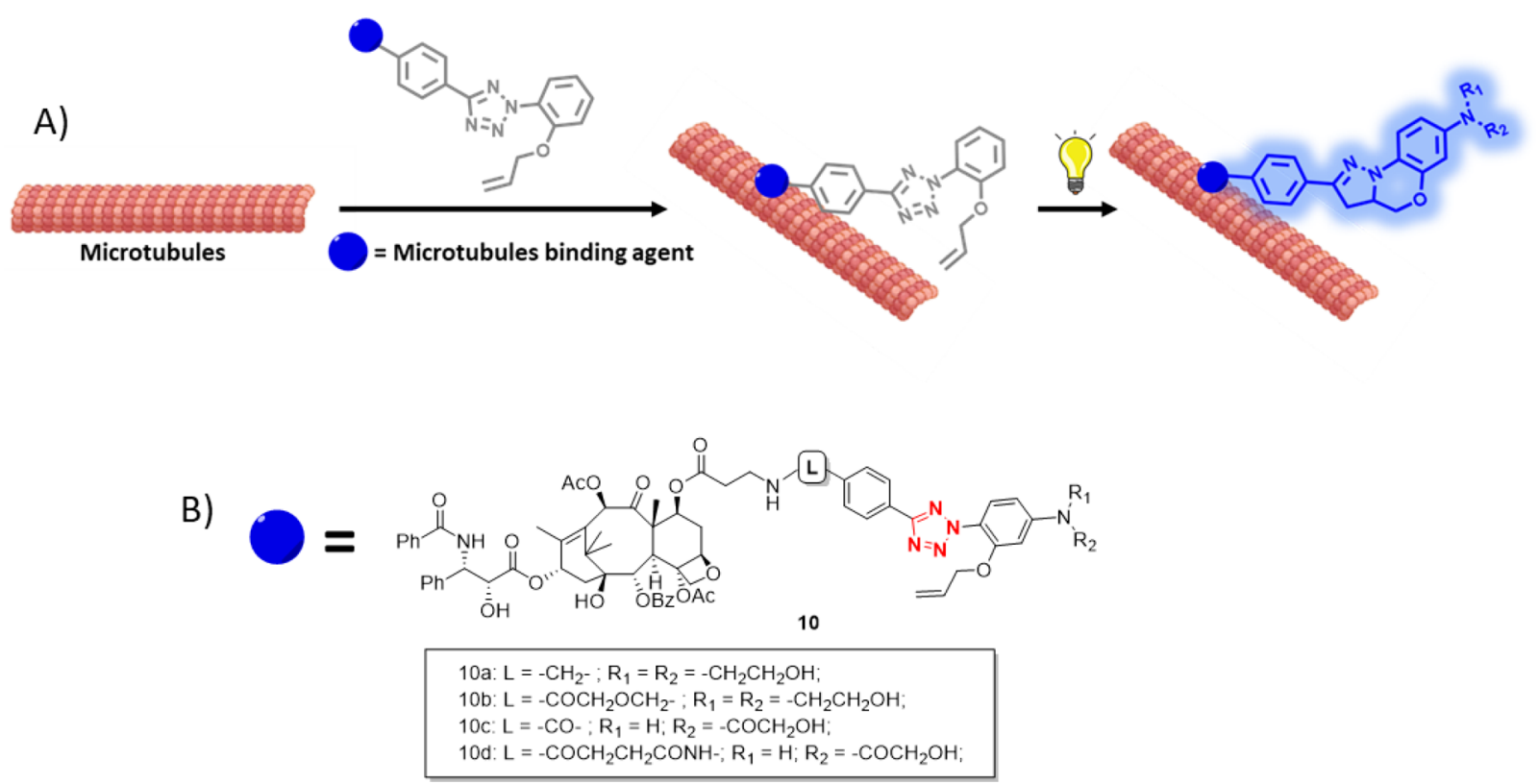

Fig. 5 A Schematic representation of microtubules detection performed with tetrazole- $\beta$-alanyltaxol conjugate. B Structures of 7- $\beta$-alanyltaxolTetrazole conjugates (10a-d) used for microtubules detection 
in para-position of C-phenyl ring. The presence of an alkene moiety on tetrazole scaffold remarkably enhanced cycloaddition rate and, upon irradiation at $302 \mathrm{~nm}$, a nearly quantitative yield of pyrazoline was achieved in only $70 \mathrm{~s}$. The authors investigated also the fluorescence of the generated product and found out that the simultaneous presence of two electron-withdrawing moieties on phenyl rings, as in the case of compound 10d (Fig. 5), determined a significant fluorescence increase of the resulting pyrazoline at $528 \mathrm{~nm}\left(\Phi_{\mathrm{f}}=0.056,112\right.$-folds higher compared to starting tetrazole). To verify the efficacy of this system, HeLa cells were treated with $5 \mu \mathrm{M}$ of preactivated 10a for $30 \mathrm{~min}$. The binding of the cycloaddition adduct to microtubules was confirmed through confocal microscopy analysis, which proved pyrazoline co-localization with Alexa Fluor-568 based immunostain for microtubules. Subsequently, they have examined the photoactivation in vivo on $\mathrm{CHO}$ cells: after incubation with $1 \mu \mathrm{M}$ of tetrazole $\mathbf{1 0 c}$, cells were irradiated with a metal halide light source (bandwidth $=300-395 \mathrm{~nm}$ ). After only $5 \mathrm{~s}$, a significant increase of emission was recorded, between 535 and $545 \mathrm{~nm}$, and a $79 \%$ yield of pyrazoline was measured. To demonstrate the elevated spatio-temporal control of the process, the experiment was repeated with 10a, using a $405 \mathrm{~nm}$ pulse laser, and irradiating only specific regions for $11 \mathrm{~s}$. As a consequence, a strong fluorescence enhancement was observed only in the illuminated areas, confirming the selectivity of the photoactivation process and the possibility to label specific regions [66].

\subsection{Nucleic acid labeling}

Recently, click chemistry has been also extensively employed for applications on nucleic acids, like oligonucleotides labeling, metabolic modification of both DNA and RNA, and optimization of biological assays (e.g. polymerase chain reaction) [11]. Because of the interesting results achieved with 2,5-diaryl tetrazoles in protein labeling, photoclick chemistry found recent application even in this field.

The first example has been reported in 2014 by Wagenknecht and his research group, which used the cycloaddition for post-synthetic modification of oligonucleotides [67]. They have modified 2-deoxyuridine to obtain the tetrazole-modified nucleotide derivative $\mathbf{1 1}$ (Fig. 6), which has been incorporated into three different oligonucleotide sequences, through solid-phase synthesis. The photoreactivity of the tetrazole-oligonucleotide conjugate $(2.5 \mu \mathrm{M})$ was tested towards a cyanine probe (sulfo-Cy3) modified with maleimide moiety. Irradiation with $365 \mathrm{~nm}$ LED led to the fast formation of the expected pyrazoline (characterized by emission at $400 \mathrm{~nm}$ ), which was more efficient with doublestranded oligonucleotides (reaching $34 \%$ yield) due to the better accessibility to tetrazole with this DNA conformation [67]. This pioneering work demonstrated not only that 2,5-diaryl tetrazoles could be easily incorporated into DNA and RNA sequences, but also that nucleotide presence does not reduce the photoreactivity of these compounds, ensuring efficient labeling.

To develop a high-performing light-up probe for the detection of nucleic acids, 2-deoxyuridine has been modified also with tetrazole 12 (Fig. 6), exploiting the elevated

A)

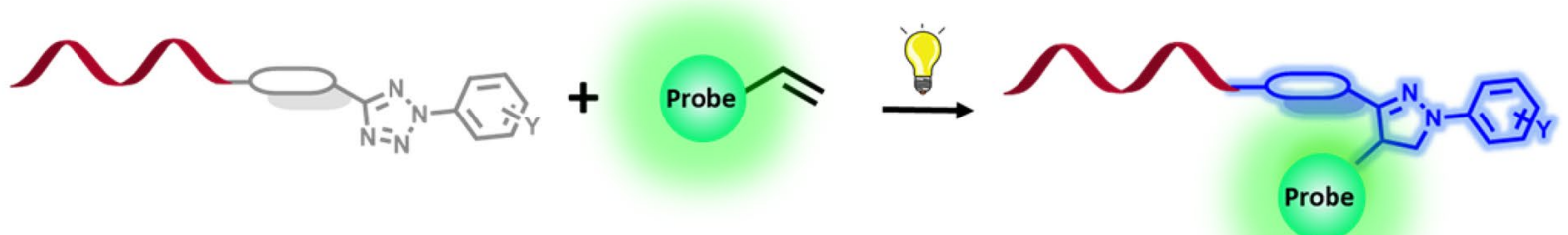

B)
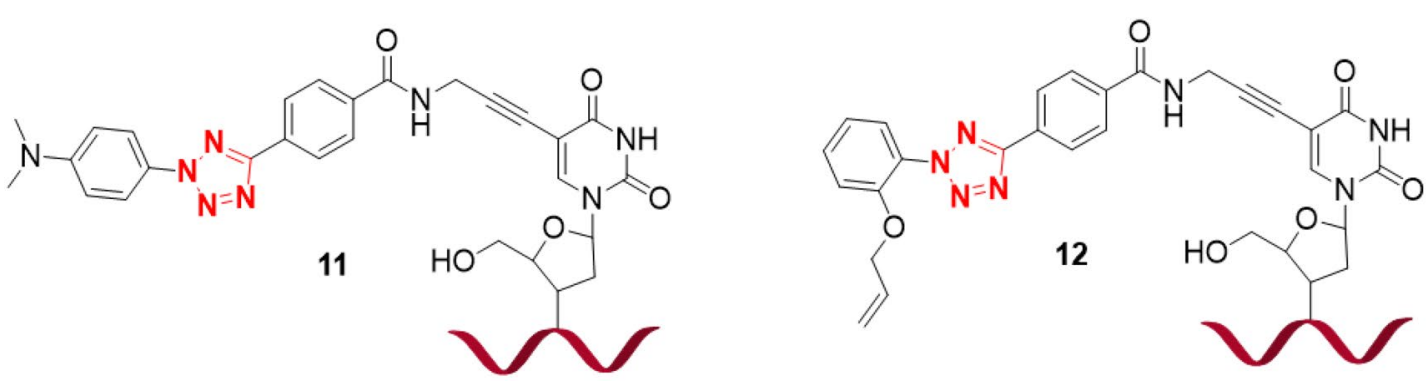

Fig. 6 A Schematic representation of photoclick labeling of tetrazole-modified oligonucleotides. B Chemical structures of the compounds $\mathbf{1 1}$ and 12 
efficiency of intramolecular photoclick reaction. The modified nucleotide was validated upon irradiation at $302 \mathrm{~nm}$. The rapid formation of pyrazoline led to a significant increase in fluorescence emission at $530 \mathrm{~nm}$. The process has been investigated also on double-stranded oligonucleotides finding out that the fluorescence intensity of the resulting pyrazoline was strictly influenced by oligonucleotide sequence and a significant quenching of the emission has been observed with duplex DNA, compared to singlestranded. Then, this interesting behavior has been exploited to detect abasic sites (AP) in nucleic acid sequences. The oligonucleotide-containing tetrazole was mixed with DNA with different contents of AP sites $(0 \%, 5 \%, 10 \%, 50 \%$, $100 \%$ ) and the resulting fluorescence light-up, recorded after one minute of exposition to a $302 \mathrm{~nm}$ lamp, was linearly correlated to AP content, evidencing the elevated sensitivity of pyrazoline fluorescence to DNA anomalies and conformational variations [68].

The possibility to efficiently modify oligonucleotide sequences with 2,5-diaryl tetrazoles expanded their applications as sensors.

Recently, two different research groups reported two elegant examples of how microRNA-tetrazole conjugates can be exploited to detect specific nucleic acid sequences in vitro and in the cellular environment. In 2016, Zhang et al., designed an innovative assay to identify target genes of specific mRNAs. They selected three cancer-related sequences,
miR-106a, miR-27, and miR-122, conjugating them to tetrazole 13 (Fig. 7). The photoreactivity of the substrates has been evaluated with methyl fumarate modified with Biotin in PBS: 5 min of irradiation at $302 \mathrm{~nm}$ led to the generation of the fluorescent product in good yields. Once they have verified that conjugation to tetrazole moiety did not alter their biological functions, miR-122 conjugated to compound $\mathbf{1 3}$ (Fig. 7) has been used to develop an innovative "Pull-Down" protocol, commonly performed using Streptavidin-coated beds to capture biomolecules tagged with Biotin [69]. In detail, HepG2 cells were transfected with tetrazole-modified miR-122 (100 nM) and the abundance of corresponding target genes was evaluated through quantitative Real-Time PCR analysis, after the addition of Biotin-methyl fumarate complex and consequent irradiation. Obtained results confirmed that the efficiency of this protocol was comparable to the commonly used biotinylation method, as both procedures provided similar relative miR-122 levels. To further validate this procedure, they have evaluated the association of miRNA with AGO2 protein, which binds microRNAs sequences and is involved in RNA-mediated gene silencing and in the assembly of RNA-induced silencing complex [70]. Western blot analysis confirmed an elevated recovery of AGO2 protein, even higher compared to the method used as a reference, probably because of stronger hydrophobic interactions between tetrazole moiety and the binding pocket of AGO2 [71]. The obtained results demonstrated

A)

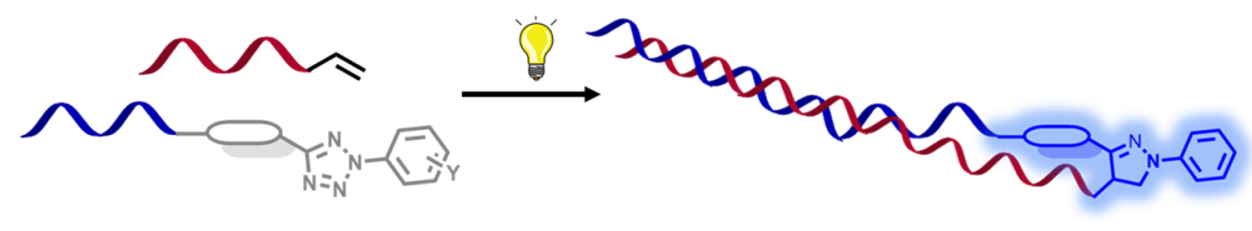

B)
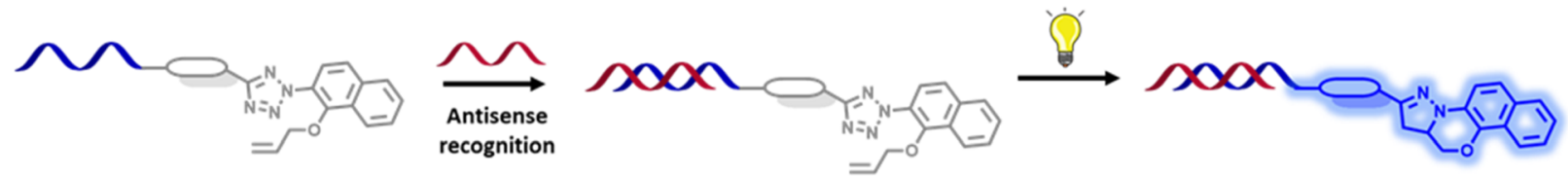

C)
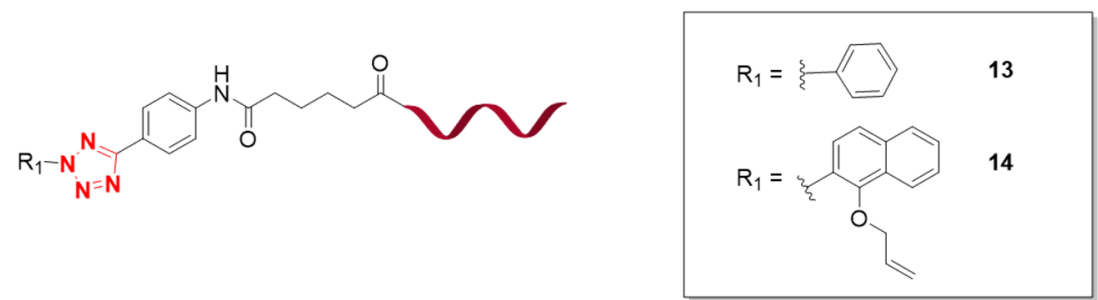

Fig. 7 A, B Schematic representation of photoclick labeling mechanisms of miRNA sequences. C Chemical structures of the compounds $\mathbf{1 3}$ and 14 
that "photoclickable" miRNAs can be efficiently employed to target specific genes in the physiological environment.

More recently, the intramolecular cycloaddition was exploited to design "photoclickable" miRNAs. Zhang et al., developed a tetrazole-modified miRNA to achieve fluorescent labeling of specific miRNAs sequences in living cells. Tetrazole 14 (Fig. 7), carrying an $o$-allyloxy group as alkene reagent, has been covalently conjugated to miR-122, using a hexanedioic acid linker. Analysis of photoreactivity highlighted fast reaction kinetic, as the complete conversion was achieved in $30 \mathrm{~s}$ in aqueous solution, and a highly fluorescent pyrazoline, whose emission increased significantly at $520 \mathrm{~nm}$ during irradiation $\left(\Phi_{\mathrm{f}}=0.25\right.$ in $\mathrm{CH}_{3} \mathrm{CN} / \mathrm{PBS}$ solution). The ability of the constructed oligonucleotide to act as a sensor in the cellular environment has been evaluated in HepG2 cells through confocal microscopy, upon excitation at $405 \mathrm{~nm}$ and $720 \mathrm{~nm}$. The presence of pyrazoline, mainly distributed into the cytoplasm, was confirmed by the intense emission recorded between 450 and $550 \mathrm{~nm}$. Finally, the efficiency of the photoclick reaction was tested irradiating HepG2 cells with a UV-lamp, and maximum fluorescence emission was achieved after only $20 \mathrm{~s}$ only in the illuminated area, demonstrating the elevated spatial and temporal control over the activation step [72].

Because of the large size of tetrazole moiety, and the necessity to shift the activation wavelength towards the visible region, the tetrazole ring was conjugated in position 5 of 2-deoxyuridine, to obtain substrate 15 (Fig. 8). This compound has been incorporated into two different oligonucleotide sequences, in an internal site, and on 5'-terminal position. The photoclick reaction has been performed among these sequences $(2.5 \mu \mathrm{M})$ and a slight excess of cyanine dye (Cy3) modified with maleimide. Upon irradiation at $365 \mathrm{~nm}$, reactions were completed after $15 \mathrm{~min}$, with product yields of $74 \%$ and $62 \%$, respectively. Resulting pyrazolines showed increasing emission between 450 and $550 \mathrm{~nm}$ and, interestingly, a fluorescence-energy transfer from this product to cyanine dye has been observed: excitation of pyrazoline chromophore resulted in an increase of the emission of Cy3 during the photoclick reaction, enabling an even more efficient detection of the product [73]. The same methodology has been applied to develop tetrazole-modified RNA oligonucleotides, to perform the photo-induced ligation with maleimide conjugated to different fluorescent dyes, as $\mathrm{Cy} 3$ and AlexaFluor555. The reaction worked efficiently, even if the yield of adduct depended on the type of probe used; higher amounts of pyrazoline were detected with AlexaFluor555 (84\%) [74]. Despite further optimizations are still required, the advantages of this approach are evident: (1) nucleobase 15 (Fig. 6) can be more easily incorporated into oligonucleotide sequence; (2) the activation can be performed with more biocompatible wavelength; and (3) the product formation can be detected through an efficient energy transfer between the pyrazoline and the cyanine.

As an alternative, to introduce smaller modifications on nucleic acid sequences, the alkene can be introduced on nucleotides. Double bonds can be easily incorporated using different approaches, not only with solid-phase synthesis but even with more sophisticated methodologies, including enzymatic approaches. To this aim, polymerases are extensively employed [75], and in the case of RNAs, methyltransferases resulted to be highly efficient with the possibility to modify only determined classes of RNA or specific sites of the sequence [76]. The results reported to date and the emerging potential of click reaction of tetrazoles with alkene further pushed the researchers to expand the pool of applications of these substrates. In 2019, Zhang and its research groups firstly described the use of tetrazoles for DNA labeling in a living system, exploiting 5-vinyl2-deoxyuridine (VdU), a modified nucleotide that can be incorporated into nucleic acid sequences of living organisms by endogenous polymerases [77]. To red-shift the activation wavelength of the substrate, they synthesized a library of tetrazoles modified in position 5 with different coumarin derivatives, responsible for a pronounced bathochromic shift, as all examined compounds displayed elevated molar absorptivity among 335 and $341 \mathrm{~nm}$. The photoreactivity of compounds has been explored on an oligonucleotide modified with acrylamide and, after 30 min of irradiation, $60.9 \%$ yield was achieved with an increase of fluorescence at $450 \mathrm{~nm}$, attributed to coumarin, as well as at $490 \mathrm{~nm}$, due to pyrazoline emission. To perform the reaction in the cellular environment, compound $\mathbf{1 6}$ (Fig. 8), characterized by an anionic sulfonyl group to facilitate nuclear permeation, has been developed. A549 cells have been incubated with VdU and $\mathbf{1 6}$ and, after $10 \mathrm{~min}$ of irradiation with $350 \mathrm{~nm}$ LED,
Fig. 8 Chemical structures of compounds 15 and 16
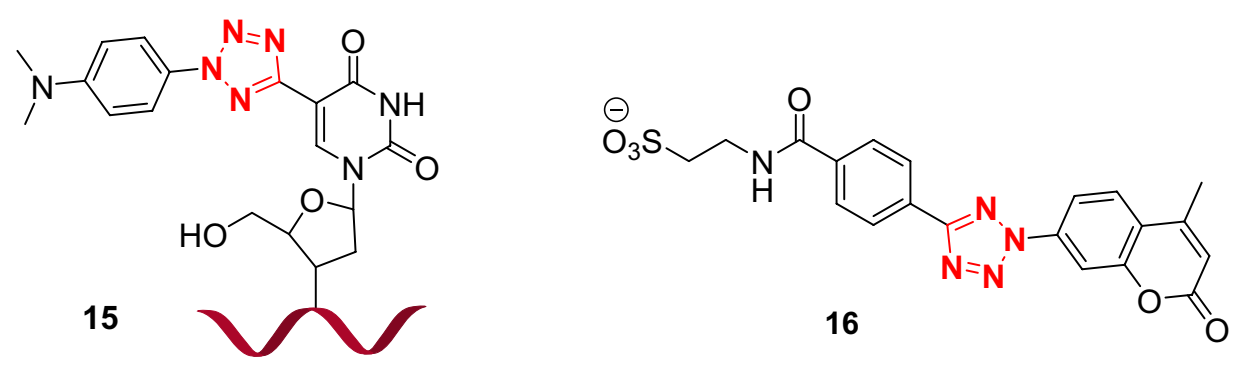
the product's formation was confirmed by intense emission detected in cell nuclei. To further demonstrate the labeling efficiency, the DNA of cells was extracted and subjected to PAGE analysis, which confirmed the incorporation of $\mathrm{VdU}$ and the formation of the expected adduct. To prove the efficacy of this approach in vivo, zebrafish embryos were treated with $\mathrm{VdU}$ and $\mathbf{1 6}$ and subsequently irradiated for $14 \mathrm{~min}$ at $350 \mathrm{~nm}$. During the experiment, a significant time-dependent fluorescence enhancement was recorded. In addition, the authors demonstrated that the whole process did not exhibit cytotoxic effects towards zebrafish, which displayed a survival rate of $80 \%$ for 40 days [78]. Additional optimizations of the reaction are still fundamental to further increase the applicability of this approach in the biological context, however, taken all together, the here described results proved the high potential of photoclick chemistry for sensing applications even in living systems.

\subsection{Photoresponsive biomaterials}

Tetrazoles resulted to be very promising scaffolds to perform biorthogonal ligation in the biological environment, as proved by the interesting examples reported to date. However, their application is not limited to the modification and labeling of proteins and nucleic acids, as deeply described before. In the last years, an increasing number of works reported the use of 2,5-diaryl tetrazoles for different purposes, including the development of innovative materials for biological applications. Recently, click reactions have been employed to prepare in situ forming hydrogels [79], polymeric materials able to incorporate a high amount of aqueous solvent and used, for example, to develop drug delivery systems or for imaging applications [80]. Zhong et al., designed an innovative hydrogel based on two distinct fourarm poly(ethylene glycol) chains: the first one conjugated to four 2,5-diaryl tetrazole groups, compound 17a (Fig. 9), and the second one to methacrylic anhydride 17b (Fig. 9), useful for protein delivery and release. Gelation of the material was performed irradiating the two substrates in a 1:1 molar ratio with a $365 \mathrm{~nm}$ lamp. The reaction proceeded rapidly, as proved by an intense increase of the emission at $460 \mathrm{~nm}$, and the activation with light enabled an accurate control over hydrogel generation. Furthermore, gel content studies showed that cross-linking was highly efficient with this process, as an elevated gel content was obtained (ranging from 85.2 to $92.7 \%$ ). Swelling experiments pointed out excellent stability, as the hydrogel resulted to be stable at physiological conditions for over 20 days. The produced material has been tested in vitro to study the release of three different proteins, CC, Ig, and rhIL-2. All of them were quantitatively

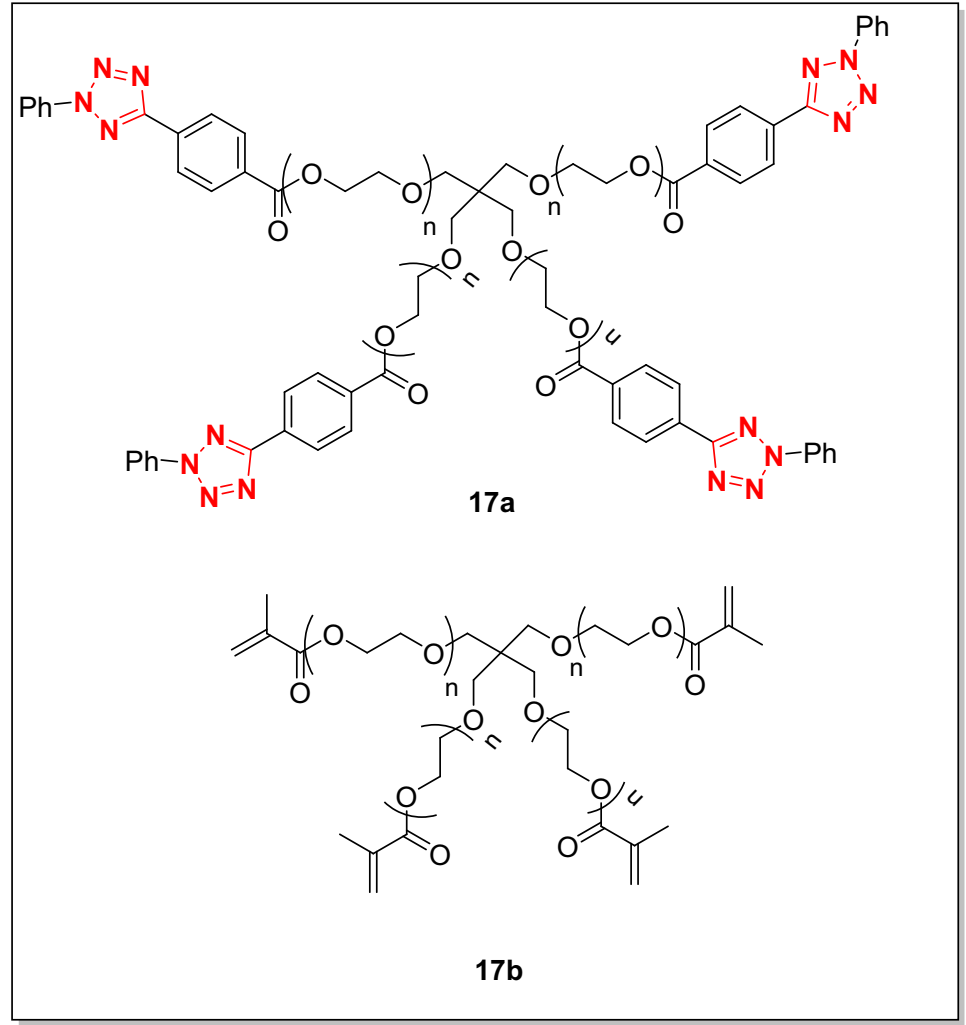

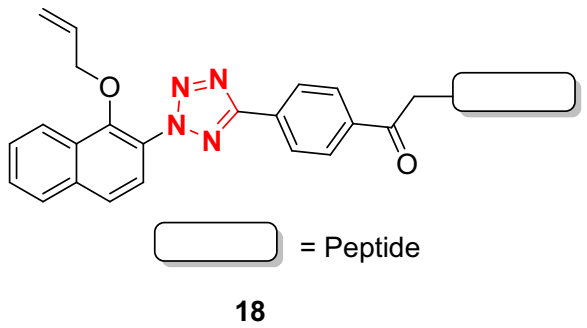

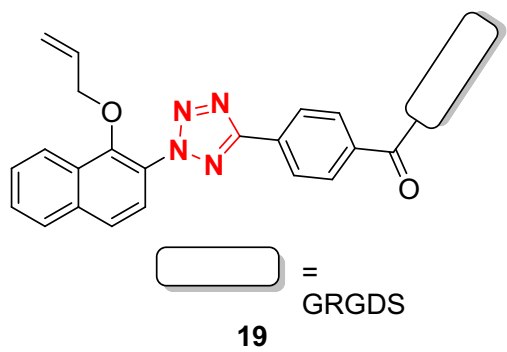

Fig. 9 Recent examples of 2,5-diaryl tetrazoles used for the development of innovative hydrogel-based materials 
released over 14-20 days and further analysis confirmed that the encapsulated proteins completely retained their biological activity, evidencing how photoclick chemistry represents a suitable method to develop biocompatible hydrogels for biomolecules delivery [81]. Similarly, to produce innovative hydrogels, Zhang et al., exploited the intramolecular cycloaddition reaction, by attaching an $o$-allyloxy moiety in position 2 of C-phenyl ring and a short peptide sequence on $\mathrm{N}$-phenyl ring, as in compound $\mathbf{1 8}$ (Fig. 9). In this case, the photoclick reaction has been used as a molecular switch to regulate the assembly and the disassembly of the material, exploiting the different aggregating properties of tetrazole and pyrazoline. The presence of tetrazole ensures the proper $\pi-\pi$ stacking interactions necessary for the assembly, balanced by hydrophilic interaction of the peptide introduced in position 4 of $N$-phenyl ring. After the identification of the most suitable peptide sequence, able to guarantee hydrogel formation, the folding properties of the structure have been investigated. After $2 \mathrm{~min}$ of irradiation at $302 \mathrm{~nm}$, quantitative pyrazoline formation was achieved and hydrogel assembly was observed. Further irradiation (over $3 \mathrm{~min}$ ) induced gel collapse, and a complete disassembly was achieved after $10 \mathrm{~min}$. In this case, the hydrogel has been used for a different purpose, i.e. as photodegradable cell culture medium, to control the cellular microenvironment upon activation with light. To test the validity of this approach, the authors evaluated the differentiation of $\mathrm{C} 2 \mathrm{C} 12$ cells induced by horse serum (HS), encapsulated into the hydrogel. In detail, HS was encapsulated into the tetrazole-based material and its release in presence of cells was induced after 2 min of UV activation. Consequently, cell differentiation was determined by immunostaining of MyoD protein, a marker of differentiation, through fluorescence microscopy. Finally, the innovative material constituted by compound $\mathbf{1 8}$ (Fig. 9) has been used to encapsulate human mesenchymal stem cells, to verify the possibility of regulating their microenvironment. Not only cells have maintained their viability after encapsulation, but UV exposure and gel photodegradation strongly influenced cell spreading and morphology, confirming the possibility to regulate cell culture medium and their consequent growth [82].

Further optimization of this strategy led to the development of a new hydrogel, based on tetrazole 18 (Fig. 9), used not only for 3D encapsulation of the cells but also for microRNAs delivery, which acquired increasing interest for their promising therapeutic potential as anticancer agents [83]. To this aim, compound $\mathbf{1 9}$ (Fig. 9) was modified with the short tripeptide sequence Arginylglycylaspartic acid (RGD), able to selectively recognize $\alpha_{v} \beta_{3}$ integrin overexpressed in cancer cells [84], plus one glycine $(G)$ and one serine (S) residues at the $N$ - and $C$-termini, respectively. The gel formed by the conjugate 19-GRGDS has been irradiated with UV-lamp and a rapid formation of expected pyrazoline was proved by significant fluorescence enhancement, accompanied by gel degradation: the sol-gel transition was completed within 5 min. Subsequent experiments also demonstrated the possibility to perform 3D cell culture into the gel, as U87 cells remained alive for 8 days after encapsulation, as well as irradiation and consequent gel degradation did not affect cells viability. The efficiency of the gel for miRNA delivery has been tested with tumor-suppressive miR-34a in U87 cells, measuring a significant increase of endogenous miR34a (35-fold). Moreover, the introduction of this specific miRNA sequence into selected cells induced a significant biological response: indeed, luciferase assay demonstrated the inhibition of the expression of several important genes targeted by miR-34a [85]. Altogether these results confirmed the high potential of photoresponsive hydrogels for biological and therapeutic applications.

More recently, photo-induced reaction of 2,5-diaryl tetrazole is emerging also with different purposes: Gabilondo et al., exploited the reaction to assemble innovative chitosanbased hydrogels, using the light-induced cycloaddition as cross-linking methods to generate the material. In this case, the reaction is used only to build the polymer, which resulted to be not only highly fluorescent but also stable in the physiological environment and highly biocompatible [86].

\section{The final challenge: how to overcome current limits}

Despite the increasing number of bio-applications reported for the light-mediated reaction of 2,5-diaryl tetrazoles with alkenes, it appears evident that the process still suffers from some important drawbacks. In general, two main issues limit the use of this reaction in the biological field: (1) the activation wavelength, and (2) the side reactions with nucleophiles. In all described works, 2,5-diaryl tetrazoles are activated by UV lamps (wavelengths ranging from 300 to $365 \mathrm{~nm}$ ), preventing their application for bioorthogonal labeling in vivo. Indeed, UV radiation can be easily absorbed by biological tissues and can induce serious damages [87], therefore, activation at higher wavelengths is necessary to develop a fully biocompatible process [88]. In addition, the well-documented ability of nitrile imine to react with natural nucleophiles reduces the overall efficiency of the cycloaddition, as the consistent presence of water, carboxylates, and thiols in the physiological environment lead to the generation of a plethora of side products that cannot be neglected.

Currently, to overcome these limits and identify valid alternatives, several strategies have been explored.

Concerning the possibility to activate the substrate at higher wavelengths, the first work developed to address this issue has been reported by Lin et al. They have synthesized a small library of tetrazole derivatives carrying different 
chromophores with absorption in the visible region, like naphthalene, coumarin, and highly conjugated aromatic systems. After a preliminary evaluation of their spectroscopic properties, they have investigated their photoreactivity in chloroform, at 302,365 , and $395 \mathrm{~nm}$, using methacrylate as a trapping agent. Coumarin-based compound 20 (Fig. 10) showed the highest reactivity and, after two hours of irradiation, led to the formation of the desired product in $89 \%$ yield [89]. The second attempt to red-shift the activation wavelength is represented by oligothiophenes. Tetrazoles were linked to bithiophene at $N$-2-position and modified with different aromatic systems on the carbon atom in position 5. Photoreactivity studies pointed out that the simultaneous presence of bithiophene and styrenyl moiety, as in substrate 21 (Fig. 10), afforded fast kinetic and high yield of the corresponding pyrazoline (97\%), upon activation with $405 \mathrm{~nm}$ laser [90]. Subsequently, the structure of these derivatives was further implemented and oligothiophene-based tetrazoles have been synthesized. Increasing the number of thiophene units on $N$-2-phenyl ring, a red-shift of the absorption maximum was observed. Then, activation of substrates has been explored using a $405 \mathrm{~nm}$ laser, in presence of monomethyl fumarate amide, in 1:1 ACN:PBS. The formation of pyrazoline has been detected after only $30 \mathrm{~s}$ of irradiation. Once the efficiency of the process was proved, tetrazole 22 (Fig. 10) has been designed to increase the solubility in physiological conditions using a negatively charged succinate moiety. Modification of the structure did not impair the reaction kinetic, as fast product formation was determined $\left(k_{2}=1299 \pm 110 \mathrm{M}^{-1} \mathrm{~s}^{-1}\right)[91]$.
Considering that conjugation to oligothiophenes pushed the absorption up to $405 \mathrm{~nm}$, which is still far from wavelengths suitable for biological applications, alternative strategies were developed. In general, tetrazole photoactivation is based on single-photon absorption. However, an interesting option to induce photoreactivity in the NIR region was represented by two-photon absorption. To this aim, authors synthesized different tetrazoles conjugated to one or two naphthalene units, characterized by welldescribed two-photon absorption properties [92]. Substrates were further functionalized with polyethylene glycol chains and carboxylic acids to improve the solubility in water. The photoreactivity of compounds has been preliminarily investigated upon activation at $365 \mathrm{~nm}$ and all of them showed an efficient generation of the corresponding pyrazoline. Subsequently, they have been irradiated in presence of acrylamide (in 1:1 ACN:PBS solution) with a tunable femtosecond-pulsed laser with the wavelength set at 750,730 , or $700 \mathrm{~nm}$, highlighting that more efficient cycloaddition was obtained irradiating at $700 \mathrm{~nm}$. Among all compounds, 23 (Fig. 10) displayed the fastest reaction kinetic, with zero-order rate constant $k_{0}=0.33 \mu \mathrm{M}^{-1} \mathrm{~s}^{-1}$ and the highest two-photon cross section (3.8 GM). The applicability of the reaction has been evaluated in vitro and in living cells. In the first case, acrylamide was incorporated into two mutants of superfolder green fluorescent protein, as $N^{\varepsilon}$-acryloyl-L-lysine. Tetrazole 23 [93] was reacted in presence of alkene-modified protein and, after irradiation with $700 \mathrm{~nm}$ femtosecond-pulsed laser for $4 \mathrm{~h}$, formation of desired pyrazoline adduct was confirmed

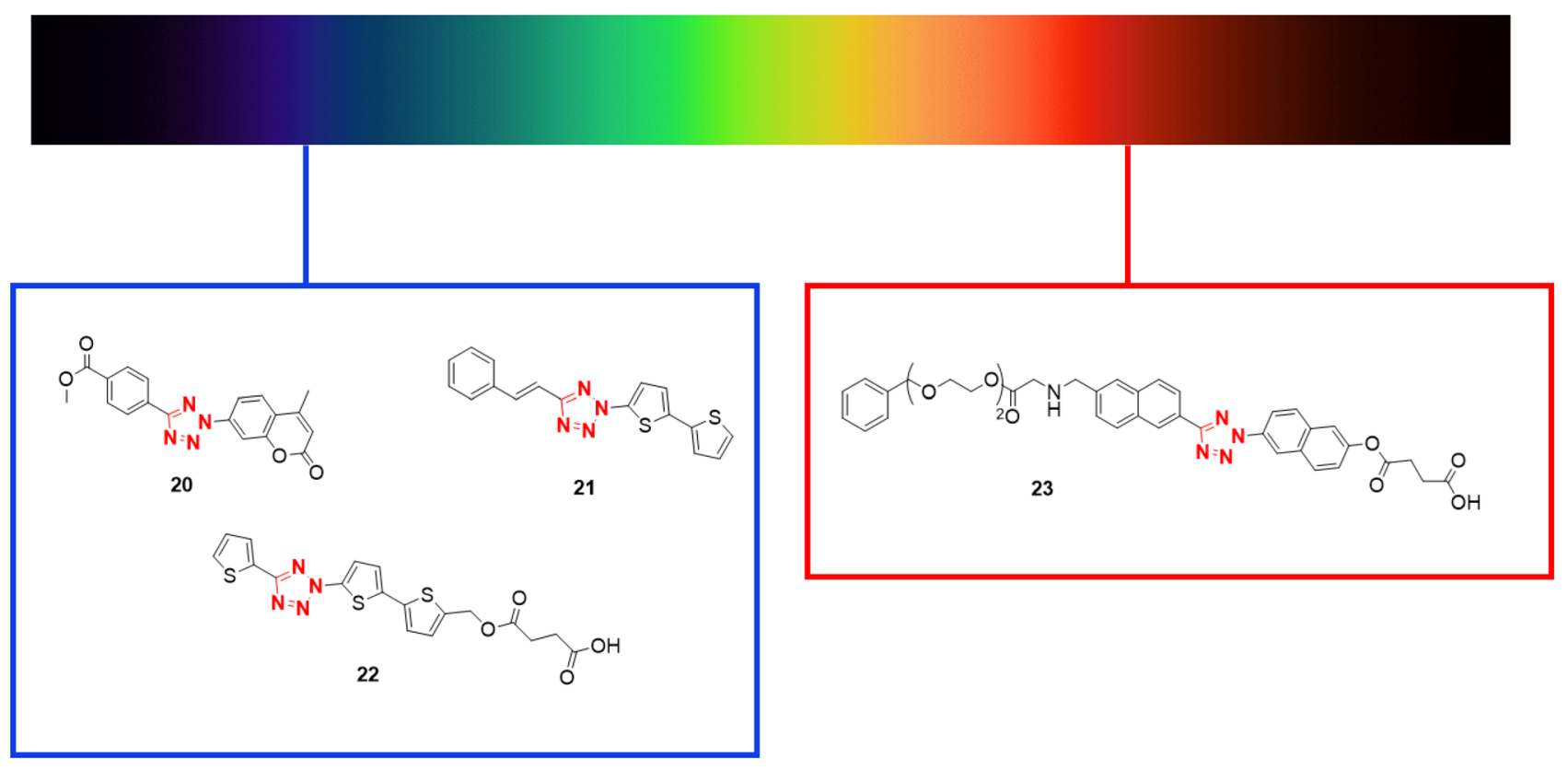

Fig. 10 Recent examples of tetrazoles activable by visible [20, 21, and 22] or NIR light [23] 
by ESI-MS analysis, with the conversion of $16.5 \%$. Conversely, the efficacy in living systems has been tested to detect microtubules with fumarate-modified docetaxel. $\mathrm{CHO}$ cells were incubated with tetrazoles $23(3 \mu \mathrm{M})$ and irradiated with a $700 \mathrm{~nm}$ laser, then the reaction was monitored through confocal laser scanning microscopy, recording the emission between 462 and $580 \mathrm{~nm}$. The maximum intensity was detected after $43 \mathrm{~s}$, only on irradiated cells, proving that two-photon activation enables an efficient photoclick reaction, even in living systems [93].

As demonstrated by reported examples, the activation wavelength can be easily modulated through the optimization of tetrazole structures. Despite the significant progresses accomplished, several modifications and optimizations of tetrazole scaffold can be performed, like (1) further expansion of the conjugated system; (2) a direct conjugation of the substrate to other probes absorbing in the NIR region; or (3) exploit non-covalent interactions for the activation, as photoactivation induced by energy transfer processes [94].

Although several alternatives have been proposed to overcome the activation wavelength issue, only a few practical solutions have been identified to reduce side reactions of nitrile imine and favor the cycloaddition reaction. Very recently, Freccero et al., reported an in-depth investigation focused on how electronic effects of substituents introduced on $\mathrm{N}$-2-phenyl and on C-5-phenyl ring can modulate the reactivity and, subsequently, cycloaddition selectivity. Briefly, they have synthesized a small library of different substituted, water-soluble, tetrazoles, to analyze nitrile imine generation efficiency and hydration vs cycloaddition selectivity. While electronic properties showed only a little influence on tetrazole photolysis, they induced significantly different reactivity towards the dipolarophile. In particular, the introduction of electron-withdrawing groups on C-5-phenyl ring and electron-donating moieties on $\mathrm{N}$-2-phenyl ring, as in compound 24 (Fig. 11) ensured quantitative yields of the corresponding pyrazoline in aqueous solution, even in presence of a low amount of alkene. However, the same study revealed that, to achieve both remarkable selectivity and high fluorescence quantum yield of the corresponding pyrazoline, the opposite configuration of substituents is more suitable, as in compound 25 (Fig. 11): this tetrazole afforded a highly emitting pyrazoline with good selectivity in water solution [95]. This interesting investigation highlighted the complexity of this photochemical process but, at the same time, it certainly contributed to elucidate tetrazoles behavior, underlining the sensitivity of the different reaction steps to the electronic nature of involved reagents.

Except for the stabilization of nitrile imine intermediate and the development of highly electron-rich substrates, other promising alternative strategies to improve reaction selectivity are still missing. For this reason, several authors suggested that this cycloaddition cannot be employed to tag proteins, since carboxylates or thiols are present on aminoacids lateral chains [96].

Conversely, Luo et al., explored the possibility to exploit carboxylates as a protein-labeling site. They have evaluated the reactivity of different substituted substrates with propionic acid, in 1:1 ACN:Buffer and found out that reactivity and product distribution were dependent on $\mathrm{pH}$ solution. In particular, nucleophilic addition to carboxylic acid was favored at acid or neutral $\mathrm{pH}$, while in basic conditions, the cycloaddition becomes the preferred pathway. Compound 26 (Fig. 11) was selected to test reaction efficiency on amino acids and, upon irradiation at $302 \mathrm{~nm}$, the formation of the desired adduct was observed in high yields (ranging from 33 to $78 \%$ ), depending on the type of amino acid, and the presence of competitive reagents did not reduce the selectivity of the process. Compound $\mathbf{2 6}$ was chosen as a model to label Histone Deacetylase 1 and 2 in HepG2 cell lysates and, after 10 min of UV irradiation, electrophoresis analysis evidenced the formation of the expected adduct [97].

Herner et al. proposed that tetrazoles could be used to develop efficient photoaffinity labels to study, for example, the preferred biological targets of new potential drugs and elucidate their mechanism of action. To validate their hypothesis, they have modified a 2,5-diaryl tetrazole through conjugation to two drugs, Dasatinib, a potent inhibitor of Bcr-Abl kinase [98], and JQ-1, a potent inhibitor of the BET family of bromodomain proteins [99]. In vitro analysis of the photoinduced reaction among modified tetrazoles, and their corresponding target proteins, showed robust cross-linking (ranging from 60 to 95\%), derived from the reaction of nitrile imine with the carboxylate of glutamate residue, as pointed out by LC-MS analysis. Authors demonstrated the efficacy of this probe also in situ, as K562 cells were incubated with the probes and, after 5 min of irradiation at $302 \mathrm{~nm}$, PAGE analysis of extracted lysate demonstrated that both probes efficiently captured the target proteins, with low off-target interactions [100]. The same year, another work highlighted that 2,5-diaryl tetrazoles could be more efficiently employed as photoaffinity labels, exploiting their reactivity towards carboxylic acids. In particular, authors developed three classes of compounds: (1) simple 2,5-diaryl tetrazoles, to test the efficiency of the reaction with carboxylates in physiological environment and protein labeling; (2) tetrazoles conjugated to well-known kinase binder STS, to test the selectivity of the process and obtain selective photolabeling of specific protein; (3) turn-on fluorescent probes, to further expand the applications of the process. STS-Tetrazole conjugates, as 27 (Fig. 11), were irradiated in presence of recombinant Protein Kinase A (PKA) for $10 \mathrm{~min}$ and the corresponding adduct was effectively detected. The process worked efficiently also in the cellular context (HepG2 cells) as selective labeling of PKA was 
<smiles>[R]c1ccc(-c2nnn(-c3ccc([R])cc3)n2)cc1</smiles>

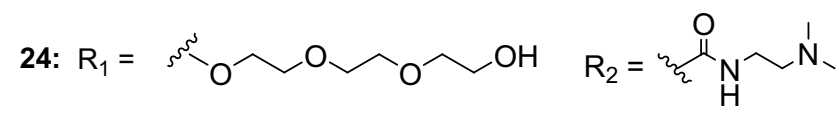

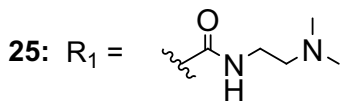

$\mathrm{R}_{2}=$ s.<smiles>C#CCOc1ccc(-c2nnn(-c3ccc(NC(=O)CCCCCCC(=O)NO)cc3)n2)cc1</smiles>

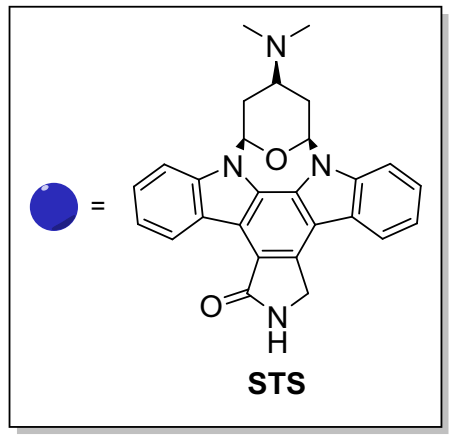<smiles>C#CCOc1ccc(-c2nnn(-c3ccc(OCC(=O)NCCNC(=O)NCC)cc3)n2)cc1</smiles>

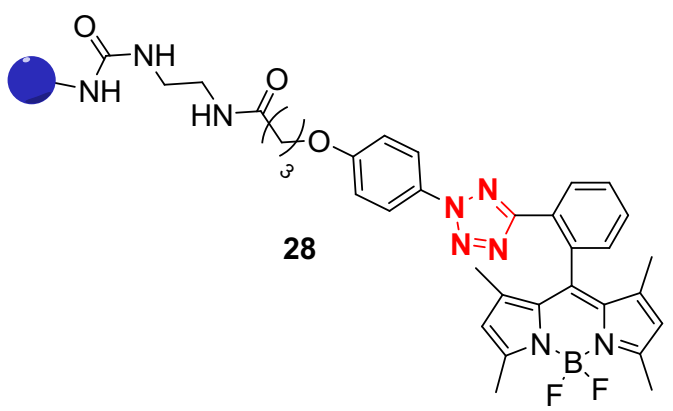<smiles>[X]CCNC(=O)c1nnn(-c2cccn2C)n1</smiles>

(29a)

(29b)

-S- $\quad(29 \mathrm{c})$

-Se- $(29 d)$

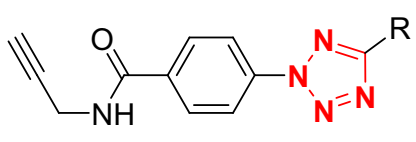

30: $\mathrm{R}=-\mathrm{Ph}$

31: $\mathrm{R}=-\mathrm{Me}$

Fig. 11 Recent examples of tetrazole scaffolds designed to optimize cycloaddition efficiency (24 and $\mathbf{2 5}$ ) and as innovative photoaffinity probes (26-31)

achieved and system performances were even higher compared to other common photoaffinity labels, as diazirines [101]. For the third class of sensors, authors exploited the ability of tetrazoles to act as fluorescence quencher: they synthesized different 2,5-diaryl tetrazoles-BODIPY conjugates whose emission was regenerated after irradiation and protein labeling. Photoactivation of compound $\mathbf{2 8}$ in water (Fig. 11) induced an 80-fold fluorescence enhancement, as a consequence of the reaction with acetic acid, and analogous behavior was observed in presence of two proteins, HSA and BSA. Use of STS-labeled compound 28 enabled the selective imaging of endogenous PKA in HepG2 cells: intense fluorescence emission was recorded in the cell cytoplasm and was persistent even after several wash cycles, demonstrating that was generated by the formation of a strong covalent bond [102].

This approach can also be exploited to study protein-protein interactions, for example using a genetically encoded tetrazole, which can be incorporated into protein sequence, as reported by Lin. They synthesized different 2-pyrroletetrazole derivatives (compounds $\mathbf{2 9}$ a-d in Fig. 11), differing for the type of heteroatom introduced in $\gamma$ position of amino acid. Photo cross-linkers were introduced on Schistosoma Japonicum glutathione-S-transferase homodimer (position E52) and obtained proteins were irradiated at $302 \mathrm{~nm}$, for $15 \mathrm{~min}$. SDS-PAGE analysis revealed the formation of the expected dimer with similar yields for all tested substrates, as a consequence of tetrazole photoactivation [103]. In this case, the authors did not employ a 2,5-diaryl 
tetrazole, but a mono-aromatic derivative; however, this type of scaffold showed activation properties comparable to previously described compounds and represents an interesting example of a genetically encoded probe that can be exploited to study protein-protein interactions in their native environment. More recently, an analogous of compound 29, has been conjugated to ubiquitin and used to monitor and clarify the activities of deubiquitinating enzymes in live cells, an important target against several diseases, upon activation at $365 \mathrm{~nm}$ [104]. In the same scenario, Hacker et al., exploited the high reactivity of 2,5-diaryl tetrazoles against carboxylates to identify new potential binding sites for inhibitors to treat bacterial infections. Taking advantage of the high content of aspartate and glutamate in the bacterial proteome, they synthesized different tetrazole-based scaffolds and investigated their photoreactivity towards Staphylococcus aureus. Compound 30 (Fig. 11) was able to react with the highest number of carboxylate residues and led to the identification of around 5000 aspartates and glutamates residues after 20 min of irradiation, performed between 280 and $315 \mathrm{~nm}$. To validate the efficacy of this approach in living bacteria, they have used 31 (Fig. 11), as it displayed higher cellular uptake. Upon irradiation, this molecule efficiently labeled 3298 residues in $S$. aureus, as proved by gel electrophoresis and mass analysis. Elevated labeling was detected also in Escherichia coli and Salmonella typhimurium, demonstrating the wide applicability of this method to map bacteria proteome. This alternative use of 2,5-diaryl tetrazoles could be particularly useful to identify novel binding sites for potential ligands with antibacterial activity [105].

Nowadays, alternative applications of 2,5-diaryl tetrazoles continue to emerge and their reactivity towards biological nucleophiles became also an appealing tool to assemble complex drugs. A very recent example of a partially new use of tetrazole compounds has been described by Holland and Fay, that developed a small library of 2-naphthalene5-aryl tetrazoles to combine photoclick chemistry with radiochemistry. Briefly, they have investigated the photochemical properties of these compounds and found out that the introduction of a methoxy group on naphthalene unit ensures efficient tetrazole photolysis at $365 \mathrm{~nm}$ and moderate yield of corresponding nucleophilic adduct (23\%). Subsequently, the tetrazole was conjugated to DFO, through an alkyl spacer, a hexadentate chelate that is used in clinical PET with ${ }^{89} \mathrm{Zr}-\mathrm{mAbs}$, able to coordinate the ${ }^{89} \mathrm{Zr}^{4+}$ metal ion, to realize compound $\mathbf{3 2}$ (Fig. 12). The photoclick reaction was exploited to conjugate this molecule to monoclonal antibody Trastuzumab, used for the treatment of different types of cancers. PET imaging studies on SK-OV-3 xenografts confirmed the efficacy of this complex conjugate, as they evidenced the high distribution of the antibody in tumor tissue, with elevated uptake and selectivity [106].

Taken together, the here described works represent important milestones in the development of more efficient photoclick reactions involving 2,5-diaryl tetrazoles. It has been demonstrated that it is possible to perform the activation at biocompatible wavelengths, through simple modifications of tetrazole scaffold. Moreover, the increasing attention of researchers contributed to fill the gap of information regarding the mechanism of this complex photochemical process, from the generation of the intermediate to addition to the alkene. Although side reactions of nitrile imine have been considered an insurmountable obstacle, recently, it has been demonstrated that the reactivity of this particular species can be easily driven towards the desired reaction pathway. Furthermore, it became clear that the reaction of nitrile imine with nucleophiles does not represent the Achille's heel of this process, but is an appealing opportunity to explore alternative and effective labeling approaches.

\section{Conclusion and outlooks}

Since 2008 [50], photoclick reaction of 2,5-diaryl tetrazoles found an increasing number of applications, especially within biological field. Compared to other common click reactions, as copper-mediated azide-cycloaddition and Inverse-Electron-Demanded Diels-Alder [2], this process presents some unique features, as the excellent stability of the substrate in the physiological environment, low toxicity, and fluorogenicity of the cycloaddition with dipolarophiles, which enables efficient sensing of a specific biomolecule.
Fig. 12 Structure of TetrazoleDFO conjugate 32 reported by Holland et al.

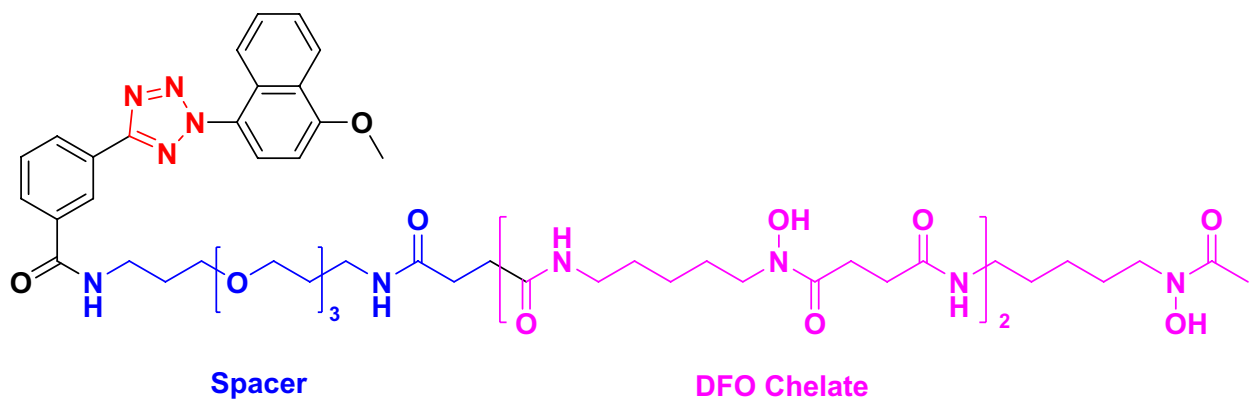

32 
The main field of bio-applications of 2,5-diaryl tetrazoles regarded mainly bioorthogonal labeling of proteins, however, they have been employed to modify nucleic acid structures and to develop innovative biomaterials, as hydrogels. Furthermore, they are emerging even in different research fields, as in material sciences, for the development of polymers needed for photoligation systems [107] or photo crosslinkers in nanoparticles [108].

Despite the several efforts dedicated to the optimization of compound structures and process parameters, some drawbacks limited the use of photoclick chemistry in the biological field. The first one is the activation wavelength restricted to the UV region, which causes severe damages to biological systems. Extended aromatic systems have been employed to shift the activation wavelength towards the near-IR region or to exploit two-photon absorption process. Important results have been accomplished following these strategies, however, further optimizations of the system are still required to perform an efficient photoinduced cycloaddition in the biological environment, especially in vivo. Based on the experimental evidences reported to date, direct conjugation of tetrazole ring to probes presenting absorption in the NIR region could be fundamental to achieve biocompatible photoactivation. Obviously, to accomplish these results, tetrazole units require consistent modification and novel structures should be rationally designed to optimize optical properties. Alternatively, other approaches can be explored and we suggest that an interesting option is represented by photocatalysis. In this context, promising results have been already obtained with upconverting nanoparticles, based on lanthanide sensitizer and activator ions, able to induce a rapid conversion of tetrazole $\left(\lambda_{\max }=346 \mathrm{~nm}\right)$ into reactive nitrile imine upon irradiation with NIR light at $974 \mathrm{~nm}$, thanks to an energy transfer between the sensitizer, that absorbs the radiation, and the activator, which emits light at different wavelengths [109]. However, to avoid the use of metal, photoactivation of tetrazole in presence of organic photosensitizers still needs to be explored.

The second major issue which affects the photoclick reaction of tetrazoles with alkenes is represented by the collateral reactions of nitrile imine with biological nucleophiles. This aspect cannot be neglected because it significantly reduces the efficiency of the process. As extensively described in the previous section, it has been demonstrated that reactivity of nitrile imine can be addressed towards the formation of the desired pyrazoline, introducing proper modifications on tetrazole scaffold, as substituents with elevated steric hindrance [61] or electron-donating groups on $\mathrm{N}$-2-phenyl ring [49]. However, the reaction of nitrile imine with nucleophiles has not been considered only an undesired outcome, but also an opportunity to explore different applications for 2,5-diaryl tetrazoles. Therefore, different research groups proposed that tetrazoles could be rather employed as photoaffinity labels exploiting the reaction with carboxylate residues of proteins [102], for studying protein-protein interactions [103] or the distribution of new potential drugs [100]. Considering all the promising results reported to date, the increasing interest in photoclick chemistry, and the high versatility of the light-generated intermediate, we envision that photoactivable 2,5-diaryl tetrazoles will become an even more powerful tool in many research fields, from biology to material sciences.

Acknowledgements A.B. was supported by an AIRC fellowship for Italy. V.P. was supported by a PostDoc fellowship type A funded by the University of Pavia. Open Access Funding is provided by the University of Pavia within the CRUI-CARE Agreement.

\section{Declarations}

Conflict of interest On behalf of all authors, the corresponding author states that there is no conflict of interest.

Open Access This article is licensed under a Creative Commons Attribution 4.0 International License, which permits use, sharing, adaptation, distribution and reproduction in any medium or format, as long as you give appropriate credit to the original author(s) and the source, provide a link to the Creative Commons licence, and indicate if changes were made. The images or other third party material in this article are included in the article's Creative Commons licence, unless indicated otherwise in a credit line to the material. If material is not included in the article's Creative Commons licence and your intended use is not permitted by statutory regulation or exceeds the permitted use, you will need to obtain permission directly from the copyright holder. To view a copy of this licence, visit http://creativecommons.org/licenses/by/4.0/.

\section{References}

1. Xi, W., Scott, T. F., Kloxin, C. J., \& Bowman, C. N. (2014). Click chemistry in materials science. Advanced Functional Materials, $24,2572-2590$.

2. Kim, E., \& Koo, H. (2019). Biomedical applications of copperfree click chemistry: In vitro, in vivo, and ex vivo. Chemical Science, 10, 7835-7851.

3. Breugst, M., \& Reissig, H.-U. (2020). The Huisgen reaction: Milestones of the 1,3-dipolar cycloaddition. Angewandte Chemie International Edition, 59, 12293-12307.

4. Lewis, W. G., Green, L. G., Grynszpan, F., Radić, Z., Carlier, P. R., Taylor, P., Finn, M. G., \& Sharpless, K. B. (2002). Click chemistry in situ: Acetylcholinesterase as a reaction vessel for the selective assembly of a femtomolar inhibitor from an array of building blocks. Angewandte Chemie International Edition, 41, 1053-1057.

5. Tasdelen, M. A., \& Yagci, Y. (2013). Light-induced click reactions. Angewandte Chemie International Edition, 52, 5930-5938.

6. Gupta, S., Ameta, C., Ameta, R., Punjabi, P. B. (2020). Chapter 2-click chemistry: A tool for green chemical organic synthesis. In Green sustainable process for chemical and environmental engineering and science. (Edited by Inamuddin, R. Boddula and A. M. Asiri), pp. 13-48. Elsevier.

7. Li, N., \& Binder, W. H. (2011). Click-chemistry for nanoparticlemodification. Journal of Materials Chemistry, 21, 16717-16734. 
8. Devaraj, N. K. (2018). The future of bioorthogonal chemistry. ACS Central Science, 4, 952-959.

9. Rossin, R., \& Robillard, M. S. (2014). Pretargeted imaging using bioorthogonal chemistry in mice. Current Opinion in Chemical Biology, 21, 161-169.

10. Boutureira, O., \& Bernardes, G. J. (2015). Advances in chemical protein modification. Chemical Reviews, 115, 2174-2195.

11. Fantoni, N. Z., El-Sagheer, A. H., \& Brown, T. (2021). A Hitchhiker's guide to click-chemistry with nucleic acids. Chemical Reviews, 121, 7122-7154.

12. Bednarek, C., Wehl, I., Jung, N., Schepers, U., \& Bräse, S. (2020). The Staudinger ligation. Chemical Reviews, 120, 4301-4354

13. Dommerholt, J., Rutjes, F., \& van Delft, F. L. (2016). Strainpromoted 1,3-dipolar cycloaddition of cycloalkynes and organic azides. Top Curr Chem (Cham), 374, 1-20.

14. Oliveira, B. L., Guo, Z., \& Bernardes, G. J. L. (2017). Inverse electron demand Diels-Alder reactions in chemical biology. Chemical Society Reviews, 46, 4895-4950.

15. António, J. P. M., Russo, R., Carvalho, C. P., Cal, P. M. S. D., \& Gois, P. M. P. (2019). Boronic acids as building blocks for the construction of therapeutically useful bioconjugates. Chemical Society Reviews, 48, 3513-3536.

16. Kumar, G. S., \& Lin, Q. (2021). Light-triggered click chemistry. Chemical Reviews, 121, 6991-7031.

17. Arumugam, S., \& Popik, V. V. (2011). Light-induced heterodiels-alder cycloaddition: A facile and selective photoclick reaction. Journal of the American Chemical Society, 133, 5573-5579.

18. Kumar, P., Joshi, C., Srivastava, A. K., Gupta, P., Boukherroub, R., \& Jain, S. L. (2016). Visible light assisted photocatalytic [3 $+2]$ azide-alkyne "click" reaction for the synthesis of 1,4-substituted 1,2,3-triazoles using a novel bimetallic $\mathrm{Ru}-\mathrm{Mn}$ complex. ACS Sustainable Chemistry \& Engineering, 4, 69-75.

19. Decuypère, E., Plougastel, L., Audisio, D., \& Taran, F. (2017). Sydnone-alkyne cycloaddition: Applications in synthesis and bioconjugation. Chemical Communications, 53, 11515-11527.

20. Lim, R. K. V., \& Lin, Q. (2010). Azirine ligation: Fast and selective protein conjugation via photoinduced azirine-alkene cycloaddition. Chemical Communications, 46, 7993-7995.

21. Murale, D. P., Hong, S. C., Haque, M. M., \& Lee, J.-S. (2017). Photo-affinity labeling (PAL) in chemical proteomics: A handy tool to investigate protein-protein interactions (PPIs). Proteome Science, 15, 1-344.

22. Fairbanks, B. D., Macdougall, L. J., Mavila, S., Sinha, J., Kirkpatrick, B. E., Anseth, K. S., \& Bowman, C. N. (2021). Photoclick chemistry: A bright idea. Chemical Reviews, 121, 6915-6990.

23. Ramil, C. P., \& Lin, Q. (2014). Photoclick chemistry: A fluorogenic light-triggered in vivo ligation reaction. Current Opinion in Chemical Biology, 21, 89-95.

24. George, J. T., \& Srivatsan, S. G. (2017). Vinyluridine as a versatile chemoselective handle for the post-transcriptional chemical functionalization of RNA. Bioconjugate Chemistry, 28, $1529-1536$

25. Li Petri, G., Spanò, V., Spatola, R., Holl, R., Raimondi, M. V., Barraja, P., \& Montalbano, A. (2020). Bioactive pyrrole-based compounds with target selectivity. European Journal of Medicinal Chemistry, 208, 112783-112795.

26. Rossi, R., \& Ciofalo, M. (2020). An updated review on the synthesis and antibacterial activity of molecular hybrids and conjugates bearing imidazole moiety. Molecules, 25, 5133-5237.

27. Aggarwal, R., \& Sumran, G. (2020). An insight on medicinal attributes of 1,2,4-triazoles. European Journal of Medicinal Chemistry, 205, 112652-112697.
28. Tassinari, M., Lena, A., Butovskaya, E., Pirota, V., Nadai, M., Freccero, M., Doria, F., \& Richter, S. N. (2018). A fragmentbased approach for the development of G-quadruplex ligands: Role of the amidoxime moiety. Molecules (Basel, Switzerland), 23, 1874-1891.

29. Benassi, A., Doria, F., \& Pirota, V. (2020). Groundbreaking anticancer activity of highly diversified oxadiazole scaffolds. International Journal of Molecular Sciences, 21, 8692-8719.

30. Doria, F., Pirota, V., Petenzi, M., Teulade-Fichou, M.-P., Verga, D., \& Freccero, M. (2018). Oxadiazole/pyridine-based ligands: A structural tuning for enhancing G-quadruplex binding. Molecules, 23, 2162-2179.

31. Jampilek, J. (2019). Heterocycles in medicinal chemistry. Molecules, 24, 3839-3842.

32. Wong, M. W., Leung-Toung, R., \& Wentrup, C. (1993). Tautomeric equilibrium and hydrogen shifts of tetrazole in the gas phase and in solution. Journal of the American Chemical Society, $115,2465-2472$.

33. Neochoritis, C. G., Zhao, T., \& Dömling, A. (2019). Tetrazoles via multicomponent reactions. Chemical Reviews, 119, 1970-2042.

34. Dhiman, N., Kaur, K., \& Jaitak, V. (2020). Tetrazoles as anticancer agents: A review on synthetic strategies, mechanism of action and SAR studies. Bioorganic \& Medicinal Chemistry, 28, 115599-115620.

35. Song, W. H., Liu, M. M., Zhong, D. W., Zhu, Y. L., Bosscher, M., Zhou, L., Ye, D. Y., \& Yuan, Z. H. (2013). Tetrazole and triazole as bioisosteres of carboxylic acid: Discovery of diketo tetrazoles and diketo triazoles as anti-HCV agents. Bioorganic \& Medicinal Chemistry Letters, 23, 4528-4531.

36. Frija, L. M. T., Cristiano, M. L. S., Gómez-Zavaglia, A., Reva, I., \& Fausto, R. (2014). Genesis of rare molecules using lightinduced reactions of matrix-isolated tetrazoles. Journal of Photochemistry and Photobiology C: Photochemistry Reviews, 18, 71-90.

37. Frija, L. M. T., Ismael, A., \& Cristiano, M. L. S. (2010). Photochemical transformations of tetrazole derivatives: Applications in organic synthesis. Molecules, 15, 3757-3774.

38. Ito, S., Tanaka, Y., Kakehi, A., \& Kondo, K.-I. (1976). A facile synthesis of 2,5-disubstituted tetrazoles by the reaction of phenylsulfonylhydrazones with arenediazonium salts. Bulletin of the Chemical Society of Japan, 49, 1920-1923.

39. Zhang, Y., Liu, W., \& Zhao, Z. K. (2014). Nucleophilic trapping nitrilimine generated by photolysis of diaryltetrazole in aqueous phase. Molecules, 19, 306-315.

40. Wang, Y., Rivera Vera, C. I., \& Lin, Q. (2007). Convenient synthesis of highly functionalized pyrazolines via mild, photoactivated 1,3-dipolar cycloaddition. Organic Letters, 9, 4155-4158.

41. Blasco, E., Sugawara, Y., Lederhose, P., Blinco, J. P., Kelterer, A.-M., \& Barner-Kowollik, C. (2017). Understanding reactivity patterns in light-induced nitrile imine mediated tetrazole-ene cycloadditions. ChemPhotoChem, 1, 159-163.

42. Abe, M., Bégué, D., Silva, H. S., Dargelos, A., \& Wentrup, C. (2018). Triplet states of tetrazoles, nitrenes, and carbenes from matrix photolysis of tetrazoles, and phenylcyanamide as a source of phenylnitrene. The Journal of Physical Chemistry A, 122, 7276-7283.

43. Wentrup, C., Fischer, S., Maquestiau, A., \& Flammang, R. (1985). Nitrile imines: Thermal generation, direct observation, and subsequent trapping. Angewandte Chemie International Edition in English, 24, 56-57.

44. Wong, M. W., \& Wentrup, C. (1993). Structure of nitrilimine: Allenic or propargylic? Journal of the American Chemical Society, 115, 7743-7746. 
45. Mawhinney, R. C., Muchall, H. M., \& Peslherbe, G. H. (2004). The electronic structure of nitrilimines revisited. Chemical Communications, 2004, 1862-1863.

46. Zheng, S.-L., Wang, Y., Yu, Z., Lin, Q., \& Coppens, P. (2009). Direct observation of a photoinduced nonstabilized nitrile imine structure in the solid state. Journal of the American Chemical Society, 131, 18036-18037.

47. Clovis, J. S., Eckell, A., Huisgen, R., \& Sustmann, R. (1967). 1.3-Dipolare cycloadditionen, XXV. Der nachweis des freien diphenylnitrilimins als zwischenstufe bei cycloadditionen. Chemische Berichte, 100, 60-70.

48. Herner, A., \& Lin, Q. (2015). Photo-triggered click chemistry for biological applications. Topics in Current Chemistry, 374, 1-31.

49. Wang, Y., Song, W., Hu, W. J., \& Lin, Q. (2009). Fast alkene functionalization in vivo by photoclick chemistry: HOMO lifting of nitrile imine dipoles. Angewandte Chemie (International ed. in English), 48, 5330-5333.

50. Song, W., Wang, Y., Qu, J., Madden, M. M., \& Lin, Q. (2008). A photoinducible 1,3-dipolar cycloaddition reaction for rapid, selective modification of tetrazole-containing proteins. Angewandte Chemie International Edition, 47, 2832-2835.

51. Karrouchi, K., Radi, S., Ramli, Y., Taoufik, J., Mabkhot, Y. N., Al-aizari, F. A., \& Ansar, M. H. (2018). Synthesis and pharmacological activities of pyrazole derivatives: A review. Molecules, 23, 134-219.

52. Remy, R., \& Bochet, C. G. (2018). Application of photoclick chemistry for the synthesis of pyrazoles via 1,3-dipolar cycloaddition between alkynes and nitrilimines generated in situ. European Journal of Organic Chemistry, 2018, 316-328.

53. Jiang, S., Wu, X., Liu, H., Deng, J., Zhang, X., Yao, Z., Zheng, Y., Li, B., \& Yu, Z. (2020). Ring-strain-promoted ultrafast diaryltetrazole-alkyne photoclick reactions triggered by visible light. ChemPhotoChem, 4, 327-331.

54. Kozma, E., \& Kele, P. (2019). Fluorogenic probes for superresolution microscopy. Organic \& Biomolecular Chemistry, 17, 215-233.

55. Song, W., Wang, Y., Qu, J., \& Lin, Q. (2008). Selective functionalization of a genetically encoded alkene-containing protein via "photoclick chemistry" in bacterial cells. Journal of the American Chemical Society, 130, 9654-9655.

56. Song, W., Wang, Y., Yu, Z., Vera, C. I. R., Qu, J., \& Lin, Q. (2010). A metabolic alkene reporter for spatiotemporally controlled imaging of newly synthesized proteins in mammalian cells. ACS Chemical Biology, 5, 875-885.

57. Wang, Y., \& Lin, Q. (2009). Synthesis and evaluation of photoreactive tetrazole amino acids. Organic Letters, 11, 3570-3573.

58. Wang, J., Zhang, W., Song, W., Wang, Y., Yu, Z., Li, J., Wu, M., Wang, L., Zang, J., \& Lin, Q. (2010). A biosynthetic route to photoclick chemistry on proteins. Journal of the American Chemical Society, 132, 14812-14818.

59. Siti, W., Khan, A. K., de Hoog, H.-P.M., Liedberg, B., \& Nallani, M. (2015). Photo-induced conjugation of tetrazoles to modified and native proteins. Organic \& Biomolecular Chemistry, 13, 3202-3206.

60. Shang, X., Lai, R., Song, X., Li, H., Niu, W., \& Guo, J. (2017). Improved photoinduced fluorogenic alkene-tetrazole reaction for protein labeling. Bioconjugate Chemistry, 28, 2859-2864.

61. An, P., Lewandowski, T. M., Erbay, T. G., Liu, P., \& Lin, Q. (2018). Sterically shielded, stabilized nitrile imine for rapid bioorthogonal protein labeling in live cells. Journal of the American Chemical Society, 140, 4860-4868.

62. An, P., \& Lin, Q. (2018). Sterically shielded tetrazoles for a fluorogenic photoclick reaction: Tuning cycloaddition rate and product fluorescence. Organic \& Biomolecular Chemistry, 16, $5241-5244$.
63. Shuai, W., Wang, G., Zhang, Y., Bu, F., Zhang, S., Miller, D. D., Li, W., Ouyang, L., \& Wang, Y. (2021). Recent progress on tubulin inhibitors with dual targeting capabilities for cancer therapy. Journal of Medicinal Chemistry, 64, 7963-7990.

64. Kakraba, S., Ayyadevara, S., Penthala, N. R., Balasubramaniam, M., Ganne, A., Liu, L., Alla, R., Bommagani, S. B., Barger, S. W., Griffin, W. S. T., Crooks, P. A., \& Shmookler Reis, R. J. (2019). A novel microtubule-binding drug attenuates and reverses protein aggregation in animal models of Alzheimer's disease. Frontiers in Molecular Neuroscience, 12, 310-323.

65. Guy, R. K., Scott, Z. A., Sloboda, R. D., \& Nicolaou, K. C. (1996). Fluorescent taxoids. Chemistry \& Biology, 3, 1021-1031.

66. Yu, Z., Ho, L. Y., \& Lin, Q. (2011). Rapid, photoactivatable turn-on fluorescent probes based on an intramolecular photoclick reaction. Journal of the American Chemical Society, 133, 11912-11915.

67. Arndt, S., \& Wagenknecht, H.-A. (2014). "Photoclick" postsynthetic modification of DNA. Angewandte Chemie International Edition, 53, 14580-14582.

68. He, Z., Chen, Y., Wang, Y., Wang, J., Mo, J., Fu, B., Wang, Z., Du, Y., \& Zhou, X. (2016). A rapidly photo-activatable light-up fluorescent nucleoside and its application in DNA base variation sensing. Chemical Communications, 52, 8545-8548.

69. Louche, A., Salcedo, S. P., \& Bigot, S. (2017). Protein-protein interactions: Pull-down assays. Methods in Molecular Biology, 1615, 247-255.

70. Müller, M., Fazi, F., \& Ciaudo, C. (2020). Argonaute proteins: From structure to function in development and pathological cell fate determination. Frontiers in Cell and Developmental Biology, 7, 360-369.

71. Li, J., Huang, L., Xiao, X., Chen, Y., Wang, X., Zhou, Z., Zhang, C., \& Zhang, Y. (2016). Photoclickable MicroRNA for the intracellular target identification of microRNAs. Journal of the American Chemical Society, 138, 15943-15949.

72. Huang, L., Chen, Y., Chen, L., Xiao, X., Wang, X., Li, J., \& Zhang, Y. (2017). Photo-clickable microRNA for in situ fluorescence labeling and imaging of microRNA in living cells. Chemical Communications, 53, 6452-6455.

73. Lehmann, B., \& Wagenknecht, H. A. (2018). Fluorogenic "photoclick" labelling of DNA using a Cy3 dye. Organic \& Biomolecular Chemistry, 16, 7579-7582.

74. Krell, K., \& Wagenknecht, H.-A. (2020). Fluorogenic and bioorthogonal modification of RNA using photoclick chemistry. Biomolecules, 10, 480-490.

75. Hocek, M. (2019). Enzymatic synthesis of base-functionalized nucleic acids for sensing, cross-linking, and modulation of protein-DNA binding and transcription. Accounts of Chemical Research, 52, 1730-1737.

76. Holstein, J. M., Stummer, D., \& Rentmeister, A. (2015). Enzymatic modification of 5'-capped RNA with a 4-vinylbenzyl group provides a platform for photoclick and inverse electron-demand Diels-Alder reaction. Chemical Science, 6, 1362-1369.

77. Rieder, U., \& Luedtke, N. W. (2014). Alkene-tetrazine ligation for imaging cellular DNA. Angewandte Chemie International Edition, 53, 9168-9172.

78. Wu, Y., Guo, G., Zheng, J., Xing, D., \& Zhang, T. (2019). Fluorogenic "Photoclick" labeling and imaging of DNA with coumarin-fused tetrazole in vivo. ACS Sens, 4, 44-51.

79. Dimatteo, R., Darling, N. J., \& Segura, T. (2018). In situ forming injectable hydrogels for drug delivery and wound repair. Advanced Drug Delivery Reviews, 127, 167-184.

80. Mahinroosta, M., Jomeh Farsangi, Z., Allahverdi, A., \& Shakoori, Z. (2018). Hydrogels as intelligent materials: A brief review of synthesis, properties and applications. Materials Today Chemistry, 8, 42-55. 
81. Fan, Y., Deng, C., Cheng, R., Meng, F., \& Zhong, Z. (2013). In situ forming hydrogels via catalyst-free and bioorthogonal "Tetrazole-Alkene" photo-click chemistry. Biomacromolecules, 14, 2814-2821.

82. He, M., Li, J., Tan, S., Wang, R., \& Zhang, Y. (2013). Photodegradable supramolecular hydrogels with fluorescence turn-on reporter for photomodulation of cellular microenvironments. Journal of the American Chemical Society, 135, 18718-18721.

83. Shah, V., \& Shah, J. (2020). Recent trends in targeting miRNAs for cancer therapy. Journal of Pharmacy and Pharmacology, 72, $1732-1749$

84. Su, C.-Y., Li, J.-Q., Zhang, L.-L., Wang, H., Wang, F.-H., Tao, Y.-W., Wang, Y.-Q., Guo, Q.-R., Li, J.-J., Liu, Y., Yan, Y.-Y., \& Zhang, J.-Y. (2020). The biological functions and clinical applications of integrins in cancers. Frontiers in Pharmacology, 11, 1435-1448.

85. Zhou, Z., Yi, Q., Xia, T., Yin, W., Kadi, A. A., Li, J., \& Zhang, Y. (2017). A photo-degradable supramolecular hydrogel for selective delivery of microRNA into 3D-cultured cells. Organic \& Biomolecular Chemistry, 15, 2191-2198.

86. Guaresti, O., Crocker, L., Palomares, T., Alonso-Varona, A., Eceiza, A., Fruk, L., \& Gabilondo, N. (2020). Light-driven assembly of biocompatible fluorescent chitosan hydrogels with self-healing ability. Journal of Materials Chemistry B, 8 , 9804-9811.

87. Pattison, D. I., \& Davies, M. J. (2006). Actions of ultraviolet light on cellular structures. EXS, 2006, 131-157.

88. East, A. K., Lucero, M. Y., \& Chan, J. (2021). New directions of activity-based sensing for in vivo NIR imaging. Chemical Science, 12, 3393-3405.

89. Yu, Z., Ho, L. Y., Wang, Z., \& Lin, Q. (2011). Discovery of new photoactivatable diaryltetrazoles for photoclick chemistry via "scaffold hopping." Bioorganic \& Medicinal Chemistry Letters, 21, 5033-5036.

90. An, P., Yu, Z., \& Lin, Q. (2013). Design and synthesis of laser-activatable tetrazoles for a fast and fluorogenic red-emitting 1,3-dipolar cycloaddition reaction. Organic Letters, 15, 5496-5499.

91. An, P., Yu, Z., \& Lin, Q. (2013). Design of oligothiophene-based tetrazoles for laser-triggered photoclick chemistry in living cells. Chemical Communications, 49, 9920-9922.

92. An, J. M., Kim, S. H., \& Kim, D. (2020). Recent advances in two-photon absorbing probes based on a functionalized dipolar naphthalene platform. Organic \& Biomolecular Chemistry, 18, 4288-4297.

93. Yu, Z., Ohulchanskyy, T. Y., An, P., Prasad, P. N., \& Lin, Q. (2013). Fluorogenic, two-photon-triggered photoclick chemistry in live mammalian cells. Journal of the American Chemical Society, 135, 16766-16769.

94. Strieth-Kalthoff, F., \& Glorius, F. (2020). Triplet energy transfer photocatalysis: Unlocking the next level. Chem, 6, 1888-1903.

95. Benassi, A., Pirota, V., Doria, F., \& Freccero, M. (2021). The quest for the right trade-off for an efficient photoclick monitoring reaction. ChemPhotoChem, 2021, 1.

96. Feng, W., Li, L., Yang, C., Welle, A., Trapp, O., \& Levkin, P. A. (2015). UV-induced tetrazole-thiol reaction for polymer

\section{Authors and Affiliations}

\section{Valentina Pirota ${ }^{1} \cdot$ Alessandra Benassi $^{1} \cdot$ Filippo Doria ${ }^{1}[$ ]}

1 Department of Chemistry, University of Pavia, Viale

Taramelli 10, Pavia, Italy conjugation and surface functionalization. Angewandte Chemie International Edition, 54, 8732-8735.

97. Zhao, S., Dai, J., Hu, M., Liu, C., Meng, R., Liu, X., Wang, C., \& Luo, T. (2016). Photo-induced coupling reactions of tetrazoles with carboxylic acids in aqueous solution: Application in protein labelling. Chemical Communications, 52, 4702-4705.

98. Shah, N. P., Tran, C., Lee, F. Y., Chen, P., Norris, D., \& Sawyers, C. L. (2004). Overriding imatinib resistance with a novel ABL kinase inhibitor. Science, 305, 399-401.

99. Filippakopoulos, P., Qi, J., Picaud, S., Shen, Y., Smith, W. B., Fedorov, O., Morse, E. M., Keates, T., Hickman, T. T., Felletar, I., Philpott, M., Munro, S., McKeown, M. R., Wang, Y., Christie, A. L., West, N., Cameron, M. J., Schwartz, B., Heightman, T. D., ... Bradner, J. E. (2010). Selective inhibition of BET bromodomains. Nature, 468, 1067-1073.

100. Herner, A., Marjanovic, J., Lewandowski, T. M., Marin, V., Patterson, M., Miesbauer, L., Ready, D., Williams, J., Vasudevan, A., \& Lin, Q. (2016). 2-Aryl-5-carboxytetrazole as a new photoaffinity label for drug target identification. Journal of the American Chemical Society, 138, 14609-14615.

101. Hill, J. R., \& Robertson, A. A. B. (2018). Fishing for drug targets: A focus on diazirine photoaffinity probe synthesis. Journal of Medicinal Chemistry, 61, 6945-6963.

102. Li, Z., Qian, L., Li, L., Bernhammer, J. C., Huynh, H. V., Lee, J.-S., \& Yao, S. Q. (2016). Tetrazole photoclick chemistry: reinvestigating its suitability as a bioorthogonal reaction and potential applications. Angewandte Chemie International Edition, 55, 2002-2006.

103. Tian, Y., \& Lin, Q. (2018). Genetic encoding of 2-aryl-5-carboxytetrazole-based protein photo-cross-linkers. Chemical Соттиnications, 54, 4449-4452.

104. Gui, W., Shen, S., \& Zhuang, Z. (2020). Photocaged cell-permeable ubiquitin probe for temporal profiling of deubiquitinating enzymes. Journal of the American Chemical Society, 142, 19493-19501.

105. Bach, K., Beerkens, B. L. H., Zanon, P. R. A., \& Hacker, S. M. (2020). Light-activatable, 2,5-disubstituted tetrazoles for the proteome-wide profiling of aspartates and glutamates in living bacteria. ACS Central Science, 6, 546-554.

106. Fay, R., \& Holland, J. P. (2021). Tuning tetrazole photochemistry for protein ligation and molecular imaging. Chemistry A European Journal, 27, 4893-4897.

107. Delafresnaye, L., Schmitt, C. W., Barner, L., \& Barner-Kowollik, C. (2019). A photochemical ligation system enabling solid-phase chemiluminescence read-out. Chemistry, 25, 12538-12544.

108. Xia, H., Gao, Y., Yin, L., Cheng, X., Wang, A., Zhao, M., Ding, J., \& Shi, H. (2019). Light-triggered covalent coupling of gold nanoparticles for photothermal cancer therapy. ChemBioChem, 20, 667-671.

109. Lederhose, P., Chen, Z., Müller, R., Blinco, J. P., Wu, S., \& Barner-Kowollik, C. (2016). Near-infrared photoinduced coupling reactions assisted by upconversion nanoparticles. Angewandte Chemie International Edition, 55, 12195-12199. 